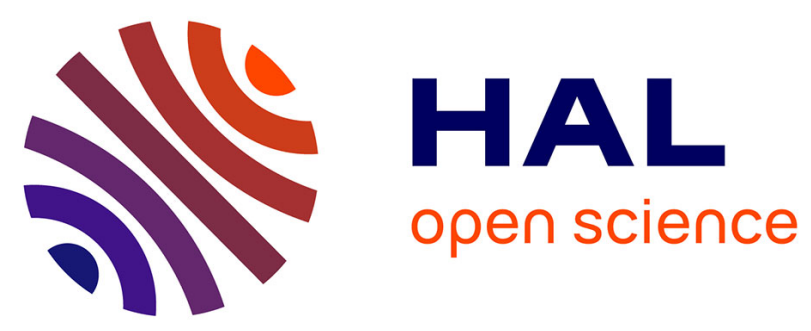

\title{
Identification of Dynamic Models in Complex Networks With Prediction Error Methods: Predictor Input Selection
}

Arne Dankers, Paul M. J. van den Hof, Xavier Bombois, Peter S. C. Heuberger

\section{- To cite this version:}

Arne Dankers, Paul M. J. van den Hof, Xavier Bombois, Peter S. C. Heuberger. Identification of Dynamic Models in Complex Networks With Prediction Error Methods: Predictor Input Selection. IEEE Transactions on Automatic Control, 2016, 61 (4), pp.937-952. 10.1109/TAC.2015.2450895 . hal-01355016

\author{
HAL Id: hal-01355016 \\ https://hal.science/hal-01355016
}

Submitted on 25 Apr 2019

HAL is a multi-disciplinary open access archive for the deposit and dissemination of scientific research documents, whether they are published or not. The documents may come from teaching and research institutions in France or abroad, or from public or private research centers.
L'archive ouverte pluridisciplinaire HAL, est destinée au dépôt et à la diffusion de documents scientifiques de niveau recherche, publiés ou non, émanant des établissements d'enseignement et de recherche français ou étrangers, des laboratoires publics ou privés. 


\title{
Identification of Dynamic Models in Complex Networks with Prediction Error Methods - Predictor Input Selection
}

\author{
Arne Dankers, Member, IEEE, Paul M. J. Van den Hof, Fellow, IEEE, Xavier Bombois and Peter S. C. \\ Heuberger
}

\begin{abstract}
This paper addresses the problem of obtaining an estimate of a particular module of interest that is embedded in a dynamic network with known interconnection structure. In this paper it is shown that there is considerable freedom as to which variables can be included as inputs to the predictor, while still obtaining consistent estimates of the particular module of interest. This freedom is encoded into sufficient conditions on the set of predictor inputs that allow for consistent identification of the module. The conditions can be used to design a sensor placement scheme, or to determine whether it is possible to obtain consistent estimates while refraining from measuring particular variables in the network. As identification methods the Direct and Two Stage Prediction-Error methods are considered. Algorithms are presented for checking the conditions using tools from graph theory.
\end{abstract}

Index Terms-System identification, closed-loop identification, graph theory, dynamic networks, linear systems.

\section{INTRODUCTION}

$\mathbf{S}$ YSTEMS IN ENGINEERING are becoming more complex and interconnected. Consider for instance, power systems, telecommunication systems, and distributed control systems. Since many of these systems form part of the foundation of our modern society, their seamless operation is paramount. However, the increasing complexity and size of the systems poses real engineering challenges (in maintaining stability of the electrical power grid, increasing data throughput of telecommunication networks, etc.). These systems cannot be operated, designed, and maintained without the help of models.

Tools from system identification are well suited to construct models using measurements obtained from a system. However, the field of system identification is primarily focused on identifying open and closed-loop systems. Recently, there has been a move to consider more complex interconnection structures. The literature on identification and dynamic networks can be split into two categories based on whether the interconnection structure of the network is assumed to be known or not. In the latter the objective is generally to detect the topology of the network, whereas in the former the focus has mainly been to identify (part of) the dynamical transfers in the network based on open-loop and closed-loop identification techniques.

The work of Arne Dankers is supported in part by the National Science and Research Council (NSERC) of Canada.

A. Dankers is with the Delft Center for Systems and Control, Delft University of Technology, adankersehifieng. com

P.M.J. Van den Hof is with the Department of Electrical Engineering, Eindhoven University of Technology, p.m.j.vandenhof@tue.nl

P.S.C. Heuberger is with the Department of Mechanical Engineering, Eindhoven University of Technology, p.s. c. heuberger@tue.n I

$\mathrm{X}$. Bombois is with Laboratoire Ampère, Ecole Centrale de Lyon (Ecully, France), xavier.bombois@ec-lyon.fr
The topology detection literature is primarily based on the methods of Granger and Granger Causality [1]. In [2], [3] it is shown that it is possible to distinguish between open and closed-loop systems (using a parametric approach). Recently, this line of reasoning has been extended to more general networks in [4], [5] (using a non-parametric approach). Several methods have appeared that automate Granger's method for detection of causal relations by using regularization terms to set certain links in the network to zero. For instance, [6], [7] directly implement an $\ell_{0}$ norm, whereas [8] uses the LASSO ([9]), and [10] uses a compressed sensing approach. In [11] a Bayesian approach for topology detection is presented. The main features that these algorithms have in common is that all internal variables in the network are assumed to be known, each internal variable is driven by an independent stochastic variable, and most papers assume all transfer functions in the network are strictly proper. Under these conditions it is shown that topology detection is possible.

Although the structure detection problem is very interesting, the underlying identification techniques, even for the case that the network structure is known, have not been fully developed yet. In particular if we consider situations that go beyond the rather restrictive conditions mentioned above. As a result, identification of (particular modules in) dynamic networks for a given interconnection structure is a relevant problem to address. Moreover, for a large number of systems in engineering the interconnection structure of the network is known (power systems, telecommunication systems etc.).

In the identification of dynamic networks attention has been given to the study of spatially distributed systems, where each node is connected only to its direct neighbors and the modules are assumed to be identical [12], [13] or not [14], [15], [16]. In these papers emphasis is on numerically fast algorithms.

In [17] closed-loop prediction-error identification methods have been extended to the situation of dynamic networks and analyzed in terms of consistency properties. The interconnection topology is very general and goes beyond the spatially distributed topology. The approach is to focus on identifying a single module embedded in a network with known interconnection structure and with general conditions on noise disturbances. Both noise and known user-defined signals (called reference signals) can drive or excite the network, while the presence of reference signals can be used to relax assumptions on the noise in the system. In the analysis of [17] it is required that all signals that directly map into the output of the considered module are taken as predictor inputs, and therefore they all need to be measured.

In this paper we consider an extension of the problem 
setting in [17]. The objective is to identify a particular module embedded in a dynamic network, and to analyze the flexibility that exists in which selection of measured variables leads to consistent identification of the module of interest. The variables that are measured are available to use as predictor inputs, i.e. variables that are used to predict the value of a particular internal variable. Specifically, the question addressed in this paper is: given a dynamic network with known interconnection structure, for which selection of predictor inputs can we guarantee that a particular module of interest can be estimated consistently?

Our approach is actually a local approach where only a limited number of variables need to be measured in order to identify the object of interest. The resulting algorithms can be applied to small to medium scale networks, or to large networks with sparse interconnection structures. It can also be used to design a sensor placement scheme tailored specifically to identifying a particular module in the network. Thus, it may be possible to avoid measuring variables that are expensive, difficult or unsafe to measure.

In order to make the step towards a selection of predictor input variables, the dynamics that appear between a selection of measured variables in a network is described in a so-called immersed network. The conditions for consistent module estimates are derived in a general context, and then specified for the Direct and Two-Stage Prediction-Error Methods, as formalized for a dynamic network case in [17]. This paper is based on the preliminary results of [18], [19] but developed and formulated here in a stronger and unifying framework, by relying predominantly on an analysis that is independent of the particular identification algorithm.

In Section II dynamic networks are defined. In Section III the prediction-error identification framework is presented, including generalizations of the Direct and Two-Stage identification methods. In Section IV an immersed network is defined as the network that is constructed by discarding nonmeasured node variables. Additionally general conditions are formulated on the predictor input variables to ensure consistent estimation of the module dynamics. In Sections V and VI the conditions on predictor inputs are specified for each identification method separately. In Section VII an algorithm based on graph theory is presented to check the conditions.

\section{System Definition And Setup}

\section{A. Dynamic Networks}

The networks that are considered in this paper are built up of $L$ elements (or nodes), related to $L$ scalar internal variables $w_{j}, j=1, \ldots, L$. It is assumed that each internal variable is such that it can be written as:

$$
w_{j}(t)=\sum_{k \in \mathcal{N}_{j}} G_{j k}^{0}(q) w_{k}(t)+r_{j}(t)+v_{j}(t)
$$

where $G_{j k}^{0}(q), k \in \mathcal{N}_{j}$ is a proper rational transfer function, $q^{-1}$ is the delay operator (i.e. $q^{-1} u(t)=u(t-1)$ ) and,

- $\mathcal{N}_{j}$ is the set of indices of internal variables with direct causal connections to $w_{j}$, i.e. $i \in \mathcal{N}_{j}$ iff $G_{j i}^{0} \neq 0$;
- $v_{j}$ is an unmeasured disturbance variable that is a stationary stochastic process with rational spectral density: $v_{j}=H_{j}^{0}(q) e_{j}$ where $e_{j}$ is a white noise process, and $H_{j}^{0}$ is a monic, stable, minimum phase transfer function;

- $r_{j}$ is an external variable that is known and can be manipulated by the user; it is an important variable that can provide deliberate (user-chosen) excitation to the network.

It may be that the disturbance and/or external variable are not present at some nodes. The entire network is defined by:

$$
\left[\begin{array}{c}
w_{1} \\
w_{2} \\
\vdots \\
w_{L}
\end{array}\right]=\left[\begin{array}{cccc}
0 & G_{12}^{0} & \cdots & G_{1 L}^{0} \\
G_{21}^{0} & 0 & \ddots & \vdots \\
\vdots & \ddots & \ddots & G_{L-1}^{0} \\
G_{L 1}^{0} & \cdots & G_{L}^{0} & 0
\end{array}\right]\left[\begin{array}{c}
w_{1} \\
w_{2} \\
\vdots \\
w_{L}
\end{array}\right]+\left[\begin{array}{c}
r_{1} \\
r_{2} \\
\vdots \\
r_{L}
\end{array}\right]+\left[\begin{array}{c}
v_{1} \\
v_{2} \\
\vdots \\
v_{L}
\end{array}\right] \text {, }
$$

where $G_{j k}^{0}$ is non-zero if and only if $k \in \mathcal{N}_{j}$ for row $j$. Using an obvious notation results in:

$$
w=G^{0} w+r+v
$$

where, $w, r$, and $v$ are vectors. If an external or disturbance variable is absent at node $i$, the $i$ th entry of $r$ or $v$ respectively is 0 . Eq. (2) is the data generating system.

There exists a path from $w_{i}$ to $w_{j}$ if there exist integers $n_{1}, \ldots, n_{k}$ such that $G_{j n_{1}}^{0} G_{n_{1} n_{2}}^{0} \cdots G_{n_{k} i}^{0}$ is non-zero. Likewise there exists a path from $r_{i}$ to $w_{j}$ (or $v_{i}$ to $w_{j}$ ) if there exist integers $n_{1}, \ldots, n_{k}$ such that $G_{j n_{1}}^{0} G_{n_{1} n_{2}}^{0} \cdots G_{n_{k} i}^{0}$ is non-zero.

The following sets will be used throughout the paper:

- $\mathcal{R}$ and $\mathcal{V}$ denote the sets of indices of all external and disturbance variables respectively present in the network.

- $\mathcal{R}_{j}$ and $\mathcal{V}_{j}$ denote the sets of indices of all the external and disturbance variables respectively with a path to $w_{j}$.

A directed graph of a dynamic network can be used to represent a network. A directed graph is a collection of nodes connected by directed edges. A directed graph of a dynamic network can be constructed as follows:

1. Let all $w_{k}, k \in\{1, \ldots, L\}$ be nodes.

2. Let all $v_{k}, k \in \mathcal{V}$ and $r_{m}, m \in \mathcal{R}$ be nodes.

3. For all $i, j \in\{1, \ldots, L\}$ if $G_{j i} \neq 0$, then add a directed edge from node $w_{i}$ to node $w_{j}$.

4. For all $k \in \mathcal{V}$ add a directed edge from $v_{k}$ to $w_{k}$.

5. For all $k \in \mathcal{R}$ add a directed edge from $r_{k}$ to $w_{k}$.

More concepts from graph theory will be used throughout the paper, but they will be presented where they are applicable. The following is an example of a dynamic network.

Example 1: Consider a network defined by:

$\left[\begin{array}{l}w_{1} \\ w_{2} \\ w_{3} \\ w_{4} \\ w_{5} \\ w_{6}\end{array}\right]=\left[\begin{array}{cccccc}0 & 0 & 0 & G_{14}^{0} & 0 & 0 \\ G_{21}^{0} & 0 & G_{23}^{0} & 0 & 0 & 0 \\ 0 & G_{32}^{0} & 0 & 0 & 0 & 0 \\ 0 & 0 & 0 & 0 & 0 & G_{46}^{0} \\ 0 & G_{52}^{0} & 0 & G_{54}^{0} & 0 & G_{56}^{0} \\ 0 & 0 & G_{63}^{0} & 0 & G_{65}^{0} & 0\end{array}\right]\left[\begin{array}{l}w_{1} \\ w_{2} \\ w_{3} \\ w_{4} \\ w_{5} \\ w_{6}\end{array}\right]+\left[\begin{array}{c}v_{1} \\ v_{2} \\ v_{3} \\ v_{4} \\ v_{5} \\ v_{6}\end{array}\right]$

shown in Fig. 1a. Its graph is shown in Fig. 1b.

All networks are assumed to satisfy the following conditions.

Assumption 1: 


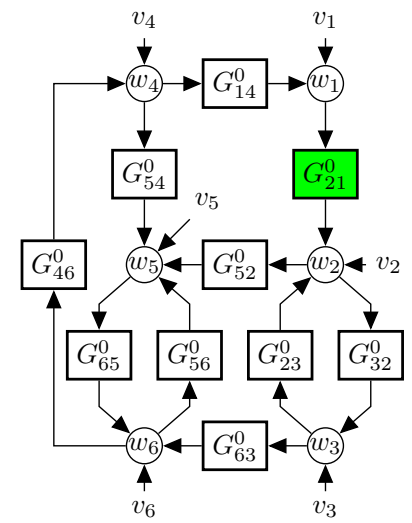

(a)

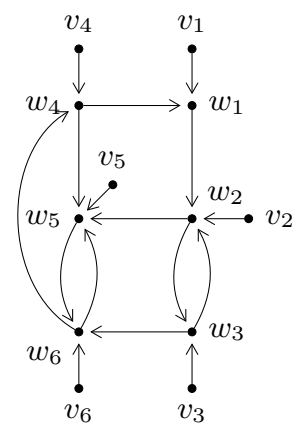

(b)
Fig. 1. A diagram (a) and graph (b) of the network for Examples 1 and 2. In (a), each rectangle represents a transfer function, and each circle represents a summation. For clarity labels of the $w_{i}$ 's have been placed inside the summations indicating that the output of the sum is the variable $w_{i}$.

(a) The network is well-posed in the sense that all principal minors of $\lim _{z \rightarrow \infty}\left(I-G^{0}(z)\right)$ are non-zero.

(b) $\left(I-G^{0}\right)^{-1}$ is stable.

(c) All $r_{m}, m \in \mathcal{R}$ are uncorrelated to all $v_{k}, k \in \mathcal{V}$. ${ }^{1}$

The well-posedness property [20] ensures that both $G^{0}$ and $\left(I-G^{0}\right)^{-1}$ only contain proper (causal) transfer functions, and still allows the occurrence of algebraic loops.

In this paper the set of internal variables chosen as predictor inputs plays an important role. For this reason, it is convenient to partition (2) accordingly. Let $\mathcal{D}_{j}$ denote the set of indices of the internal variables that are chosen as predictor inputs. Let $\mathcal{Z}_{j}$ denote the set of indices not in $\{j\} \cup \mathcal{D}_{j}$, i.e. $\mathcal{Z}_{j}=\{1, \ldots, L\} \backslash\left\{\{j\} \cup \mathcal{D}_{j}\right\}$. Let $w_{\mathcal{D}}$ denote the vector $\left[\begin{array}{lll}w_{k_{1}} & \cdots & w_{k_{n}}\end{array}\right]^{T}$, where $\left\{k_{1}, \ldots, k_{n}\right\}=\mathcal{D}_{j}$. Let $r_{\mathcal{D}}$ denote the vector $\left[r_{k_{1}} \cdots r_{k_{n}}\right]^{T}$, where $\left\{k_{1}, \ldots, k_{n}\right\}=\mathcal{D}_{j}$, and where the $\ell$ th entry is zero if $r_{\ell}$ is not present in the network (i.e. $\ell \notin \mathcal{R}$ ). The vectors $w_{\mathcal{Z}}, v_{\mathcal{D}}, v_{\mathcal{Z}}$ and $r_{\mathcal{Z}}$ are defined analogously. The ordering of the elements of $w_{\mathcal{D}}, v_{\mathcal{D}}$, and $r_{\mathcal{D}}$ is not important, as long as it is the same for all vectors. The transfer function matrix between $w_{\mathcal{D}}$ and $w_{j}$ is denoted $G_{j \mathcal{D}}^{0}$. The other transfer function matrices are defined analogously. By this notation, the network equations (2) are rewritten as:

$$
\left[\begin{array}{c}
w_{j} \\
w_{\mathcal{D}} \\
w_{\mathcal{Z}}
\end{array}\right]=\left[\begin{array}{ccc}
0 & G_{j \mathcal{D}}^{0} & G_{j \mathcal{Z}}^{0} \\
G_{\mathcal{D} j}^{0} & G_{\mathcal{D D}}^{0} & G_{\mathcal{D Z}}^{0} \\
G_{\mathcal{Z} j}^{0} & G_{\mathcal{Z D}}^{0} & G_{\mathcal{Z Z}}^{0}
\end{array}\right]\left[\begin{array}{c}
w_{j} \\
w_{\mathcal{D}} \\
w_{\mathcal{Z}}
\end{array}\right]+\left[\begin{array}{c}
v_{j} \\
v_{\mathcal{D}} \\
v_{\mathcal{Z}}
\end{array}\right]+\left[\begin{array}{c}
r_{j} \\
r_{\mathcal{D}} \\
r_{\mathcal{Z}}
\end{array}\right],
$$

where $G_{\mathcal{D D}}^{0}$ and $G_{\mathcal{Z Z}}^{0}$ have zeros on the diagonal.

\section{PREDICTION ERROR IDENTIFICATION AND EXTENSION TO DYNAMIC NETWORKS}

In this section, the prediction-error framework is presented with a focus on using the techniques in a network setting. It is an identification framework based on the one-step-ahead predictor model [21].

\footnotetext{
${ }^{1}$ Throughout this paper $r$ uncorrelated to $v$ will mean that the crosscorrelation function $R_{r v}(\tau)$ is zero for all $\tau$.
}

\section{A. Prediction Error Identification}

Let $w_{j}$ denote the variable which is to be predicted, i.e. it is the output of the module of interest. The predictor inputs are those (known) variables that will be used to predict $w_{j}$. The sets $\mathcal{D}_{j}$ and $\mathcal{P}_{j}$ are used to denote the sets of indices of the internal and external variables respectively that are chosen as predictor inputs - $w_{k}$ is a predictor input iff $k \in \mathcal{D}_{j}$, and $r_{k}$ is a predictor input iff $k \in \mathcal{P}_{j}$. The one-step-ahead predictor for $w_{j}$ is then [21]:

$$
\begin{aligned}
\hat{w}_{j}(t \mid t- & 1, \theta)=H_{j}^{-1}(q, \theta)\left(\sum_{k \in \mathcal{D}_{j}} G_{j k}(q, \theta) w_{k}(t)\right. \\
& \left.+\sum_{k \in \mathcal{P}_{j}} F_{j k}(q, \theta) r_{k}(t)\right)+\left(1-H_{j}^{-1}(q, \theta)\right) w_{j}(t)
\end{aligned}
$$

where $H_{j}(q, \theta)$ is a monic noise model, $G_{j k}(\theta)$ models the dynamics between $w_{k}$ to $w_{j}, k \in \mathcal{D}_{j}$, and $F_{j k}(q, \theta)$ models the dynamics between $r_{k}$ to $w_{j}, k \in \mathcal{P}_{j}$. The importance of including $F_{j k}(q, \theta)$ will become evident later in the paper. The prediction error is then:

$$
\begin{aligned}
& \varepsilon_{j}(t, \theta)=w_{j}(t)-\hat{w}_{j}(t \mid t-1, \theta) \\
& \quad=H_{j}(\theta)^{-1}\left(w_{j}-\sum_{k \in \mathcal{D}_{j}} G_{j k}(\theta) w_{k}-\sum_{k \in \mathcal{P}_{j}} F_{j k}(\theta) r_{k}\right)
\end{aligned}
$$

where arguments $q$ and $t$ have been dropped for notational clarity. The parameterized transfer functions $G_{j k}(\theta), k \in \mathcal{D}_{j}$, $F_{j k}(\theta), k \in \mathcal{P}_{j}$, and $H_{j}(\theta)$ are estimated by minimizing the sum of squared (prediction) errors:

$$
V_{j}(\theta)=\frac{1}{N} \sum_{t=0}^{N-1} \varepsilon_{j}^{2}(t, \theta) .
$$

where $N$ is the length of the data set. Let $\hat{\theta}_{N}$ denote the minimizer of (6). Under standard (weak) assumptions ([21]) $\hat{\theta}_{N} \rightarrow \theta^{*}$ with probability 1 as $N \rightarrow \infty$ where

$$
\theta^{*}=\arg \min _{\theta \in \Theta} \overline{\mathbb{E}}\left[\varepsilon_{j}^{2}(\cdot, \theta)\right] \text { and } \quad \overline{\mathbb{E}}:=\lim _{N \rightarrow \infty} \frac{1}{N} \sum_{t=0}^{N-1} \mathbb{E},
$$

and $\mathbb{E}$ is the expected value operator [21]. The function $\overline{\mathbb{E}}\left[\varepsilon_{j}^{2}(t, \theta)\right]$ is denoted $\bar{V}_{j}(\theta)$. If $G_{j k}\left(q, \theta^{*}\right)=G_{j k}^{0}$ the module transfer is estimated consistently.

As in closed-loop identification, identification in networks may have the problem that the disturbance affecting the "output" $w_{j}$ is correlated to one or more of the predictor inputs. In the closed-loop identification literature several methods have been developed to deal with this problem such as the Direct and Two Stage Methods [22], [23], [24]. Both methods have been extended to a network setting [17]. Generalizations of both methods to allow for a flexible choice of predictor inputs are presented in the following subsections.

\section{B. The Direct Method}

The Direct Method for identifying $G_{j i}^{0}(q)$ is defined by the following algorithm.

Algorithm 1: Direct Method.

1. Select $w_{j}$ as the output variable to be predicted. 
2. Choose the internal and external variables to include as predictor inputs (choose $\mathcal{D}_{j}$ and $\mathcal{P}_{j}$ ).

3. Construct the predictor (4).

4. Obtain estimates $G_{j k}\left(q, \hat{\theta}_{N}\right), k \in \mathcal{D}_{j}, F_{j k}\left(q, \hat{\theta}_{N}\right), k \in \mathcal{P}_{j}$ and $H_{j}\left(q, \hat{\theta}_{N}\right)$ by minimizing the sum of squared prediction errors (6).

In [17] Step 2 of the algorithm is replaced by a fixed choice, namely, $\mathcal{D}_{j}=\mathcal{N}_{j}$, and $\mathcal{P}_{j}=\emptyset$.

\section{Two Stage Method}

In the Two Stage Method, the predictor inputs are not internal variables, but projections of internal variables. The projection of $w_{k}$ onto an external variable $r_{k}$ is defined as follows. Any variable $w_{k}$ can be written as:

$$
w_{k}=\sum_{m \in \mathcal{R}_{k}} F_{k m}^{0} r_{m}+\sum_{m \in \mathcal{V}_{k}} H_{k m}^{0} v_{m} .
$$

where $F_{k m}^{0}$ and $H_{k m}^{0}$ are proper stable transfer functions. Let $w_{k}^{\left(r_{m}\right)}:=F_{k m}^{0} r_{m}$. The term $w_{k}^{\left(r_{m}\right)}$ is the projection of $w_{k}$ onto causally time shifted versions of $r_{m}$ (referred to as simply the projection of $w_{k}$ onto $r_{m}$ ). If there are more external variables available, then $w_{k}$ can be projected onto a set of external variables $r_{m}, m \in \mathcal{T}_{j}$, which is denoted by

$$
w_{k}^{\left(\mathcal{T}_{j}\right)}:=\sum_{m \in \mathcal{T}_{j}} w_{k}^{\left(r_{m}\right)}=\sum_{m \in \mathcal{T}_{j}} F_{k m}^{0} r_{m} .
$$

An estimate of $w_{k}^{\left(\mathcal{T}_{j}\right)}$ can be obtained by estimating $F_{k m}^{0}$, $m \in \mathcal{T}_{j}$ (using a Prediction-Error Method for instance) using a parametrized model $F_{k m}(q, \gamma)$ with $\gamma$ a parameter vector, resulting in an estimated model $F_{k m}\left(q, \hat{\gamma}_{N}\right)$. This model is used to generate the simulated signal:

$$
\hat{w}_{k}^{\left(\mathcal{T}_{j}\right)}\left(\hat{\gamma}_{N}\right)=\sum_{m \in \mathcal{T}_{j}} F_{k m}\left(q, \hat{\gamma}_{N}\right) r_{m}(t) .
$$

The Two Stage Method is defined as follows.

Algorithm 2: Two Stage Method.

1. Select $w_{j}$ as the output variable to be predicted.

2. Choose the external variables to project onto (choose $\mathcal{T}_{j}$ ).

3. Choose the internal and external variables to include as predictor inputs (choose $\mathcal{D}_{j}$ and $\mathcal{P}_{j}$ ).

4. Obtain estimates $\hat{w}_{k}^{\left(\mathcal{T}_{j}\right)}$ of $w_{k}^{\left(\mathcal{T}_{j}\right)}$ for each $k \in \mathcal{D}_{j}$.

5. Construct the predictor

$$
\hat{w}_{j}(t \mid t-1, \theta)=\sum_{k \in \mathcal{D}_{j}} G_{j k}(\theta) \hat{w}_{k}^{\left(\tau_{j}\right)}+\sum_{k \in \mathcal{P}_{j}} F_{j k}(\theta) r_{k} .
$$

6. Obtain estimates $G_{j k}\left(q, \hat{\theta}_{N}\right), k \in \mathcal{D}_{j}$ and $F_{j k}\left(q, \hat{\theta}_{N}\right), k \in$ $\mathcal{P}_{j}$ by minimizing the sum of squared prediction errors (6).

This algorithm is a generalization of the one in [17].

Remark 1: In Step 5 of the algorithm a noise model is optional. For simplicity it is not included in (9).

\section{CONSISTENT IDENTIFICATION ON THE BASIS OF A SUBSET OF PREDICTOR INPUT VARIABLES}

When only a subset of all node variables in a network is available from measurements, a relevant question becomes: what are the dynamical relationships between the nodes in this subset of measured variables? In Section IV-A it is shown that when only a selected subset of internal variables is considered, the dynamic relationships between these variables can be described by an immersed network. Several properties of the immersed network are investigated. In Section IV-B it is shown under which conditions the dynamics that appear between two internal variables remain invariant when reducing the original network to the immersed one. In Section IV-C the results of identification in networks are characterized. It is shown that it is the dynamics of the modules in the immersed network that are being identified, and conditions for consistency of general identification results are formulated. The results presented in this section are independent of an identification method.

\section{A. The Immersed Network}

In this subsection, we show that there exists a unique dynamic network consisting only of a given subset of internal variables, that still exactly describes the dynamics between the selected variables. Moreover, we show that this network can be constructed by applying an algorithm from graph theory for constructing an immersed graph. Given the selected variables $w_{k}, k \in\{j\} \cup \mathcal{D}_{j}$, the remaining variables $w_{n}, n \in \mathcal{Z}_{j}$ are sequentially removed from the network.

The following proposition shows that there is a unique characterization of the dynamics between the selected variables.

Proposition 1: Consider a dynamic network as defined in Section II-A that satisfies Assumption 1. Consider the set of internal variables $\left\{w_{k}\right\}, k \in \mathcal{D}_{j} \cup\{j\}$. There exists a network:

$$
\left[\begin{array}{l}
w_{j}(t) \\
w_{\mathcal{D}}(t)
\end{array}\right]=\breve{G}^{0}\left(q, \mathcal{D}_{j}\right)\left[\begin{array}{l}
w_{j}(t) \\
w_{\mathcal{D}}(t)
\end{array}\right]+\breve{F}^{0}\left(q, \mathcal{D}_{j}\right)\left[\begin{array}{l}
r_{j}(t)+v_{j}(t) \\
r_{\mathcal{D}}(t)+v_{\mathcal{D}}(t) \\
r_{\mathcal{Z}}(t)+v_{\mathcal{Z}}(t)
\end{array}\right],
$$

where $\breve{G}^{0}$ and $\breve{F}^{0}$ are unique transfer matrices of the form (using a notation analogous to that of (3)):

$$
\breve{G}^{0}=\left[\begin{array}{cc}
0 & \breve{G}_{j \mathcal{D}}^{0} \\
\breve{G}_{\mathcal{D} j}^{0} & \breve{G}_{\mathcal{D D}}^{0}
\end{array}\right] \quad \text { and } \quad \breve{F}^{0}=\left[\begin{array}{ccc}
\breve{F}_{j j}^{0} & 0 & \breve{F}_{j \mathcal{Z}}^{0} \\
0 & \breve{F}_{\mathcal{D D}}^{0} & \breve{F}_{\mathcal{D Z}}^{0}
\end{array}\right] \text {, }
$$

where $\breve{G}_{\mathcal{D D}}^{0}$ has zeros on the diagonal, $\breve{F}_{\mathcal{D D}}^{0}$ is diagonal, and if there is an index $\ell$ such that both $v_{\ell}$ and $r_{\ell}$ are not present, then the corresponding column of $\breve{F}^{0}$ is set to all zeros.

See Appendix X-A for the proof. Proposition 1 is in line with the result of [25] where conditions have been formulated for the existence of a unique interconnection matrix $\breve{G}^{0}$ on the basis of a transfer function from external inputs to node signals. The conditions in [25] typically reflect that enough entries of $\breve{G}^{0}$ and $\breve{F}^{0}$ are known (or set to zero as in our case). Proposition 1 is formulated for a particular structure, that matches with our dynamic network setup. Enforcing $\breve{G}^{0}$ to have zeros on the diagonal results in a network that does not have any "self-loops", i.e. no paths that enter and leave the same node. This matches the assumptions imposed on the data 

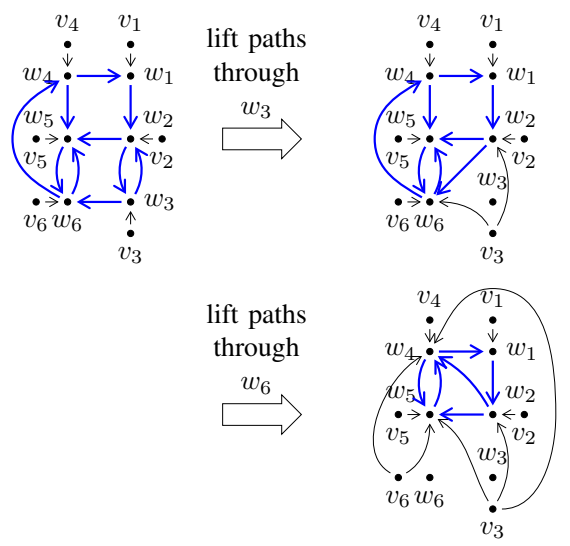

Fig. 2. Example of constructing an immersion graph. In Step 1 internal variable $w_{3}$ is removed, and in step 2 variable $w_{6}$. Edges between $w$ 's have been emphasized in thick black lines since these connections define the interconnection structure of the corresponding dynamic network.

generating system (2). Enforcing the leading square matrix of $\breve{F}^{0}$ to be diagonal results in a network where each $r_{k}$, $k \in \mathcal{D}_{j} \cup\{j\}$ only has a path to the corresponding internal variable $w_{k}$ (matching the interconnection structure of (2)). The effect of the remaining external variables is encoded in $F_{j \mathcal{Z}}^{0}$ and $F_{\mathcal{D Z}}^{0}$ without any pre-defined zero entries.

Denote the noise in (10) as:

$$
\left[\begin{array}{c}
\breve{v}_{j} \\
\breve{v}_{\mathcal{D}}
\end{array}\right]=\left[\begin{array}{cc}
\breve{F}_{j j}^{0} & 0 \\
0 & \breve{F}_{\mathcal{D D}}^{0}
\end{array}\right]\left[\begin{array}{c}
v_{j} \\
v_{\mathcal{D}}
\end{array}\right]+\left[\begin{array}{c}
\breve{F}_{j \mathcal{Z}}^{0} \\
\breve{F}_{\mathcal{D Z}}^{0}
\end{array}\right] v_{\mathcal{Z}} .
$$

Then by the Spectral Factorization Theorem [26], there exists a unique, monic, stable, minimum phase spectral factor $\breve{H}^{0}$ :

$$
\left[\begin{array}{l}
\breve{v}_{j} \\
\breve{v}_{\mathcal{D}}
\end{array}\right]=\left[\begin{array}{cc}
\breve{H}_{j j}^{0} & \breve{H}_{j \mathcal{D}}^{0} \\
\breve{H}_{\mathcal{D} j}^{0} & \breve{H}_{\mathcal{D D}}^{0}
\end{array}\right]\left[\begin{array}{l}
\breve{e}_{j} \\
\breve{e}_{\mathcal{D}}
\end{array}\right] .
$$

where $\left[\breve{e}_{j} \breve{e}_{\mathcal{D}}^{T}\right]^{T}$ is a white noise process.

In the following text it is shown that a network of the form (10) can be constructed using ideas from graph theory.

In graph theory, one way to remove nodes from a graph is by constructing an immersed graph. A graph $\mathbb{G}^{\prime}$ is an immersion of $\mathbb{G}$ if $\mathbb{G}^{\prime}$ can be constructed from $\mathbb{G}$ by lifting pairs of adjacent edges and then deleting isolated nodes [27]. Lifting an edge is defined as follows. Given three adjacent nodes $a$, $b, c$, connected by edges $a b$ and $b c$, the lifting of path $a b c$ is defined as removing edges $a b$ and $b c$ and replacing them with the edge $a c$. In Fig. 2 an immersed graph of the network of Example 1 is constructed by first removing the node $w_{3}$ and connecting $v_{3}$ to $w_{2}$ and $w_{6}$, and subsequently removing $w_{6}$ and connecting $v_{6}$ to $w_{5}$ and $w_{4}$.

In this way an immersed network can be constructed by an algorithm that manipulates the dynamics of the network iteratively. To keep track of the changes in the transfer functions iteratively, let $G_{m n}^{(i)}$ and $F_{m n}^{(i)}$ denote the transfer functions of the direct connections $w_{n}$ to $w_{m}$ and from $r_{n}$ and $v_{n}$ to $w_{m}$, respectively, at iteration $i$ of the algorithm.

Algorithm 3: Constructing an immersed network.

1. Initialize. Start with the original network:

- $G_{m n}^{(0)}=G_{m n}^{0}$ for all $m, n \in\{1, \ldots, L\}$, and

- $F_{k k}^{(0)}=1$, for all $k \in \mathcal{R} \cup \mathcal{V}, F_{m n}^{(0)}=0$ otherwise.
2. Remove each $w_{k}, k \in \mathcal{Z}_{j}$ from the network, one at a time. Let $d=\operatorname{card}\left(\mathcal{Z}_{j}\right)$. Let $\mathcal{Z}_{j}=\left\{k_{1}, \ldots, k_{d}\right\}$.

for $i=1: d$

(a) Let $\mathcal{I}_{k_{i}}$ denote the set of internal variables with edges to $w_{k_{i}}$. Let $\mathcal{O}_{k_{i}}$ denote the set of nodes with edges from $w_{k_{i}}$. Lift all paths $w_{n} \rightarrow w_{k_{i}} \rightarrow w_{m}, n \in \mathcal{I}_{k_{i}}$, $m \in \mathcal{O}_{k_{i}}$. The transfer function of each new edge from $w_{n} \rightarrow w_{m}$ is $G_{m n}^{(i)}=G_{m k_{i}}^{(i-1)} G_{k_{i} n}^{(i-1)}$

(b) Let $\mathcal{I}_{k_{i}}^{r}$ denote the set of external or disturbance variables with edges to $w_{k_{i}}$. Lift all paths $r_{n} \rightarrow w_{k_{i}} \rightarrow$ $w_{m}, n \in \mathcal{I}_{k_{i}}^{r}, m \in \mathcal{O}_{k_{i}}$. The transfer function for each new edge from $r_{n} \rightarrow w_{m}$ is $F_{n m}^{(i)}=F_{n k_{i}}^{(i-1)} G_{k_{i} n}^{(i-1)}$.

(c) If there are multiple edges between two nodes, merge the edges into one edge. The transfer function of the merged edge is equal to the sum of the transfer functions of the edges that are merged.

(d) remove the node $w_{k_{i}}$ from the network.

end

3. Remove all self-loops from the network. If node $w_{m}$ has a self loop, then divide all the edges entering $w_{m}$ by $(1-$ $\left.G_{m m}^{(d)}(q)\right)$ (i.e. one minus the loop transfer function).

Let $\breve{G}^{i^{0}}$ and $\breve{F}^{i^{0}}$ denote the final transfer matrices of the immersed network.

Remark 2: Algorithm 3 has a close connection to Mason's Rules [28], [29]. However, Mason was mainly concerned with the calculation of the transfer function from the sources (external and noise variables) to a sink (internal variable). This is equivalent to obtaining the immersed network with $\mathcal{D}_{j}=\emptyset$, i.e. all internal variables except one are removed. Importantly, Algorithm 3 is an iterative algorithm which allows for easy implementation (even for large networks), whereas Mason's rules are not iterative and complicated even for small networks.

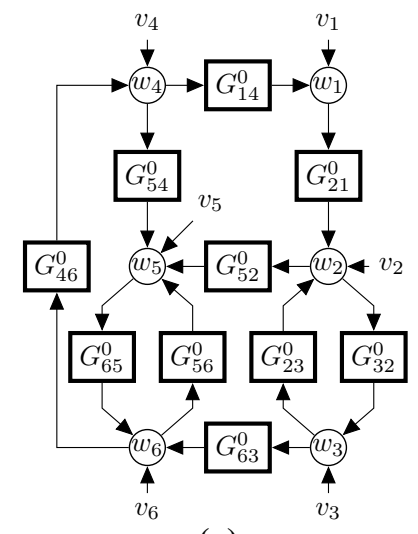

(a)

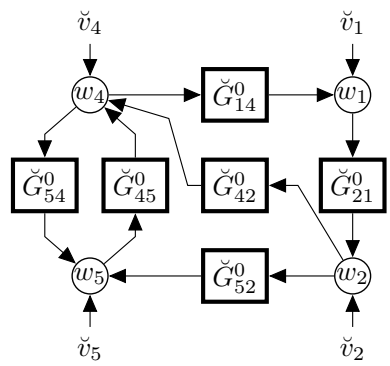

(b)
Fig. 3. (a) Original dynamic network considered in Example 2. (b) Immersed network with $w_{3}$ and $w_{6}$ removed.

Example 2: Consider the dynamic network shown in Figure 3a. The graph of this network is shown in the first graph of Fig. 2. Suppose $w_{3}$ and $w_{6}$ are to be removed from the network (i.e. $\mathcal{Z}_{j}=\{3,6\}$ ). By Algorithm 3 the network shown in Figure $3 \mathrm{~b}$ 
results. The transfer functions of the immersed network are:

$$
\begin{gathered}
\breve{G}^{i^{0}}\left(q, \mathcal{D}_{j}\right)=\left[\begin{array}{ccccc}
0 & 0 & G_{14}^{0} & 0 \\
\frac{G_{21}^{0}}{1-G_{23}^{0} G_{32}^{0}} & 0 & 0 & 0 \\
0 & G_{32}^{0} G_{46}^{0} G_{63}^{0} & 0 & G_{46}^{0} G_{65}^{0} \\
0 & \frac{G_{52}^{0}+G_{56}^{0} G_{63}^{0} G_{32}^{0}}{1-G_{56}^{0} G_{65}^{0}} & \frac{G_{54}^{0}}{1-G_{56}^{0} G_{65}^{0}} & 0
\end{array}\right] \\
\breve{F}^{i^{0}}\left(q, \mathcal{D}_{j}\right)=\left[\begin{array}{cccccc}
1 & 0 & 0 & 0 & 0 & 0 \\
0 & \frac{1}{1-G_{23}^{0} G_{32}^{0}} & 0 & 0 & \frac{G_{23}^{0}}{1-G_{23}^{0} G_{32}^{0}} & 0 \\
0 & 0 & 1 & 0 & G_{46}^{0} G_{63}^{0} & G_{46}^{0} \\
0 & 0 & 0 & \frac{1}{1-G_{56}^{0} G_{65}^{0}} & \frac{G_{56}^{0} G_{63}^{0}}{1-G_{56}^{0} G_{65}^{0}} & \frac{G_{56}^{0}}{1-G_{56}^{0} G_{65}^{0}}
\end{array}\right] .
\end{gathered}
$$

Note that the immersed network (shown in Figure $3 \mathrm{~b}$ ) is represented by the last graph shown in Figure 2.

Interestingly, the matrix $\breve{F}^{i^{0}}$ in Example 2 has the same structure as that of $\breve{F}^{0}$ in Proposition 1 . This alludes to a connection between the network characterized in (10) and immersed networks as defined by Algorithm 3 .

Proposition 2: The matrices $\breve{G}^{0}$ and $\breve{F}^{0}$ of the network characterized by $(10)$ and the matrices $\breve{G}^{i^{0}}$ and $\breve{F}^{i^{0}}$ defined by Algorithm 3 are the same.

The proof is in Appendix X-B. Since, by Proposition 2 the matrices in (10) are the same as those of the immersed network, the superscript $i$ will be dropped from this point on in the matrices defined by Algorithm 3. An important consequence of Proposition 2 is that (by Proposition 1) the immersed network is unique.

Instead of calculating the matrices of the immersed network iteratively, it is also possible to derive analytic expressions for the matrices $\breve{G}^{0}$ and $\breve{F}^{0}$.

Proposition 3: Consider a dynamic network as defined in (2) that satisfies Assumption 1. For a given set $\{j\} \cup \mathcal{D}_{j}$ the transfer function matrices $\breve{G}^{0}$ and $\breve{F}^{0}$ of the immersed network are: $^{2}$

$$
\begin{aligned}
& {\left[\begin{array}{cc}
0 & \breve{G}_{j \mathcal{D}}^{0} \\
\breve{G}_{\mathcal{D} j}^{0} & \breve{G}_{\mathcal{D D}}^{0}
\end{array}\right]=\left[\begin{array}{c}
1-\tilde{G}_{j j} \\
I-\operatorname{diag}\left(\tilde{G}_{\mathcal{D D}}^{0}\right)
\end{array}\right]^{-1}\left[\begin{array}{cc}
0 & \tilde{G}_{j \mathcal{D}}^{0} \\
\tilde{G}_{\mathcal{D} j}^{0} & \tilde{G}_{\mathcal{D D}}^{0}-\operatorname{diag}\left(\tilde{G}_{\mathcal{D D}}^{0}\right)
\end{array}\right]} \\
& {\left[\begin{array}{ccc}
\breve{F}_{j j}^{0} & 0 & \breve{F}_{j \mathcal{Z}}^{0} \\
0 & \breve{F}_{\mathcal{D D}}^{0} & \breve{F}_{\mathcal{D Z}}^{0}
\end{array}\right]=\left[\begin{array}{cc}
1-\tilde{G}_{j j} \\
I-\operatorname{diag}\left(\tilde{G}_{\mathcal{D D}}^{0}\right)
\end{array}\right]^{-1}\left[\begin{array}{ccc}
1 & 0 & \tilde{F}_{j \mathcal{Z}}^{0} \\
0 & I & \tilde{F}_{\mathcal{D Z}}^{0}
\end{array}\right]}
\end{aligned}
$$

where

$$
\begin{aligned}
{\left[\begin{array}{cc}
\tilde{G}_{j j} & \tilde{G}_{j \mathcal{D}} \\
\tilde{G}_{\mathcal{D} j} & \tilde{G}_{\mathcal{D D}}
\end{array}\right] } & =\left[\begin{array}{cc}
0 & G_{j \mathcal{D}}^{0} \\
G_{\mathcal{D} j}^{0} & G_{\mathcal{D D}}^{0}
\end{array}\right]+\left[\begin{array}{c}
G_{j \mathcal{Z}}^{0} \\
G_{\mathcal{D Z}}^{0}
\end{array}\right]\left(I-G_{\mathcal{Z Z}}^{0}\right)^{-1}\left[\begin{array}{ll}
G_{\mathcal{Z} j}^{0} & G_{\mathcal{Z D}}^{0}
\end{array}\right], \\
{\left[\begin{array}{c}
\tilde{F}_{j \mathcal{Z}} \\
\tilde{F}_{\mathcal{D} \mathcal{Z}}
\end{array}\right] } & =\left[\begin{array}{c}
G_{j \mathcal{Z}}^{0} \\
G_{\mathcal{D Z}}^{0}
\end{array}\right]\left(I-G_{\mathcal{Z Z}}^{0}\right)^{-1} .
\end{aligned}
$$

The proof is in Appendix X-C. The transfer functions $\tilde{G}_{m n}$ correspond to $G_{m n}^{(d)}$ in Step 3 of Algorithm 3 .

The immersed network inherits some useful properties from the original network.

Lemma 1: Consider a dynamic network as defined in (2) that satisfies Assumption 1 and a given set $\{j\} \cup \mathcal{D}_{j}$.

1. Consider the paths from $w_{n}$ to $w_{m}, n, m \in \mathcal{D}_{j}$ that pass only through nodes $w_{\ell}, \ell \in \mathcal{Z}_{j}$ in the original network. If

\footnotetext{
${ }^{2}$ The arguments $q$ or $\mathcal{D}_{j}$ (or both) of $\breve{G}_{j k}^{0}\left(q, \mathcal{D}_{j}\right)$ and $\breve{F}_{j k}^{0}\left(q, \mathcal{D}_{j}\right)$ are sometimes dropped for notational clarity.
}

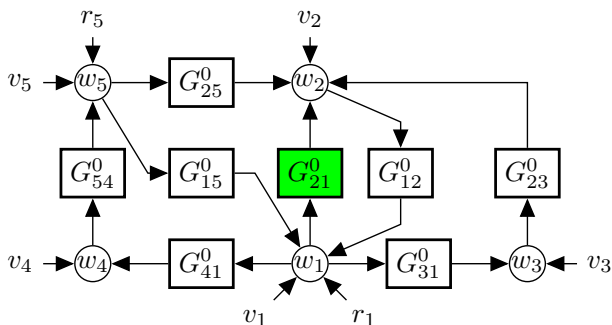

Fig. 4. Network analyzed in Examples 3 and 7.

all these paths and $G_{m n}^{0}(q)$ have a delay (are zero), then $\breve{G}_{m n}^{0}\left(q, \mathcal{D}_{j}\right)$ has a delay (is zero).

2. Consider the paths from $r_{n}$ to $w_{m}$ (or $v_{n}$ to $w_{m}$ ), $n \in \mathcal{Z}_{j}$, $m \in \mathcal{D}_{j}$. If all these paths pass through at least one node $w_{\ell}, \ell \in \mathcal{D}_{j}$ then $\breve{F}_{m n}^{0}\left(q, \mathcal{D}_{j}\right)=0$.

For a proof see Appendix X-D.

\section{B. Conditions to Ensure $\breve{G}_{j i}^{0}\left(q, \mathcal{D}_{j}\right)=G_{j i}^{0}(q)$}

A central theme in the previous section was that the transfer function $\breve{G}_{j i}^{0}\left(\mathcal{D}_{j}\right)$ in the immersed network may not be the same as the transfer function $G_{j i}^{0}$ in the original network. In other words, by selecting a subset of internal variables to be taken into account, the dynamics between two internal variables might change. In this section conditions are presented under which the module of interest, $G_{j i}^{0}$, remains unchanged in the immersed network, i.e. $\breve{G}_{j i}^{0}\left(q, \mathcal{D}_{j}\right)=G_{j i}^{0}(q)$.

The following two examples illustrate two different phenomena related to the interconnection structure that can cause the dynamics $\breve{G}_{j i}^{0}\left(q, \mathcal{D}_{j}\right)$ to be different from $G_{j i}^{0}(q)$.

Example 3: Consider the dynamic network

$\left[\begin{array}{c}w_{1} \\ w_{2} \\ w_{3} \\ w_{4} \\ w_{5}\end{array}\right]=\left[\begin{array}{ccccc}0 & G_{12}^{0} & 0 & 0 & G_{15}^{0} \\ G_{21}^{0} & 0 & G_{23}^{0} & 0 & G_{25}^{0} \\ G_{31}^{0} & 0 & 0 & 0 & 0 \\ G_{41}^{0} & 0 & 0 & 0 & 0 \\ 0 & 0 & 0 & G_{54}^{0} & 0\end{array}\right]\left[\begin{array}{c}w_{1} \\ w_{2} \\ w_{3} \\ w_{4} \\ w_{5}\end{array}\right]+\left[\begin{array}{c}v_{1}+r_{1} \\ v_{2} \\ v_{3} \\ v_{4} \\ v_{5}+r_{5}\end{array}\right]$.

shown in Fig. 4. The objective of this example, is to choose $\mathcal{D}_{2}$ such that in the immersed network $\breve{G}_{21}^{0}\left(\mathcal{D}_{2}\right)=G_{21}^{0}$ (denoted in green). A key feature of the interconnection structure in this example is that there are multiple paths from $w_{1}$ to $w_{2}$ : $w_{1} \rightarrow w_{2}, w_{1} \rightarrow w_{3} \rightarrow w_{2}, w_{1} \rightarrow w_{4} \rightarrow w_{5} \rightarrow w_{2}$, etc.

Start by choosing $\mathcal{D}_{2}=\{1\}$, then by Proposition 3 ,

$$
\breve{G}_{21}^{0}(q,\{1\})=G_{21}^{0}(q)+G_{23}^{0}(q) G_{31}^{0}(q)+G_{25}^{0}(q) G_{54}^{0}(q) G_{41}^{0}(q) \text {. }
$$

Two of the terms comprising this transfer function correspond to the two paths from $w_{1}$ to $w_{2}$ that pass only through $w_{k}, k \in$ $\mathcal{Z}_{2}\left(\mathcal{Z}_{2}=\{3,4,5\}\right)$. From Algorithm 3 this is not surprising since the paths $G_{23}^{0} G_{31}^{0}$ and $G_{25}^{0} G_{54}^{0} G_{41}^{0}$ must be lifted to remove the nodes $w_{3}, w_{4}$ and $w_{5}$ from the original network. Clearly, for this choice of $\mathcal{D}_{2}, \breve{G}_{21}^{0}\left(\mathcal{D}_{2}\right) \neq G_{21}^{0}$.

Now choose $\mathcal{D}_{2}=\{1,5\}$. By Proposition 3

$$
\breve{G}_{21}^{0}(q,\{1,5\})=G_{21}^{0}(q)+G_{23}^{0}(q) G_{31}^{0}(q) .
$$

Again, one of the terms comprising $\breve{G}_{21}^{0}(q,\{1,5\})$ corresponds to the (only) path from $w_{1}$ to $w_{2}$ that passes only through $w_{k}$, $k \in \mathcal{Z}_{2}\left(\mathcal{Z}_{2}=\{3,4\}\right)$. 
Finally, choose $\mathcal{D}_{2}=\{1,3,5\}$. By Proposition 3 $\breve{G}_{21}^{0}(q,\{1,3,5\})=G_{21}^{0}(q)$ as desired. Note that for this choice of $\mathcal{D}_{2}$ every path except $G_{21}^{0}$ from $w_{2}$ to $w_{1}$ is "blocked" by a node in $\mathcal{D}_{2}$.

In general, one internal variable $w_{k}$ from every independent path $w_{i}$ to $w_{j}$ must be included in $\mathcal{D}_{j}$ to ensure that $\breve{G}_{j i}^{0}\left(q, \mathcal{D}_{j}\right)=G_{j i}^{0}(q)$. This is proved later in Proposition 4.

However, before presenting the proposition, there is a second phenomenon related to the interconnection structure of the network that can cause the dynamics $\breve{G}_{j i}^{0}\left(q, \mathcal{D}_{j}\right)$ to be different from $G_{j i}^{0}(q)$, as illustrated in the next example.

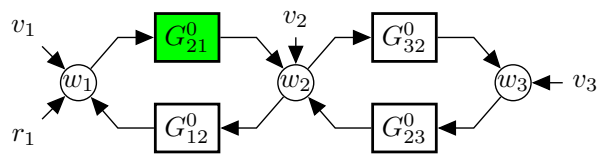

Fig. 5. Network that is analyzed in Example 4.

Example 4: Consider the network shown in Fig. 5. The objective of this example, is to choose $\mathcal{D}_{2}$ such that in the immersed network $G_{21}^{0}\left(\mathcal{D}_{2}\right)=G_{21}^{0}$ (denoted in green).

Note that in this network there is only one independent path from $w_{1}$ to $w_{2}$. Choose $\mathcal{D}_{2}=\{1\}$. By Proposition 3

$$
\breve{G}_{21}^{0}(q,\{1\})=\frac{G_{21}^{0}(q)}{1-G_{23}^{0}(q) G_{32}^{0}(q)}
$$

which is not equal to $G_{21}^{0}(q)$ as desired. The reason the factor $\frac{1}{1-G_{23}^{0} G_{32}^{0}}$ appears is because when lifting the path $G_{23} G_{32}$ a self-loop from $w_{2}$ to $w_{2}$ results. Thus, in step 3 of Algorithm 3 the transfer functions of the edges coming into $w_{2}$ are divided by the loop transfer function.

For the choice $\mathcal{D}_{2}=\{1,3\}, \breve{G}_{21}^{0}(\{1,3\})=G_{21}^{0}$ as desired. Note that in for this choice of $\mathcal{D}_{2}$, all paths from $w_{2}$ to $w_{2}$ are "blocked" by a node in $\mathcal{D}_{2}$.

In general, if $\mathcal{D}_{j}$ is chosen such that no self-loops from $w_{j}$ to $w_{j}$ result due to the lifting of the paths when constructing the immersed network, the denominator in Step 3 of Algorithm 3 is reduced to 1 . From these two examples we see that:

- Every parallel path from $w_{i}$ to $w_{j}$ should run through an input in the predictor model, and

- Every loop on the output $w_{j}$ should run through an input in the predictor model.

This is formalized in the following proposition.

Proposition 4: Consider a dynamic network as defined in Section II-A that satisfies Assumption 1. The transfer function $\breve{G}_{j i}^{0}\left(q, \mathcal{D}_{j}\right)$ in the immersed network is equal to $G_{j i}^{0}(q)$ if $\mathcal{D}_{j}$ satisfies the following conditions:

(a) $i \in \mathcal{D}_{j}, j \notin \mathcal{D}_{j}$,

(b) every path $w_{i}$ to $w_{j}$, excluding the path $G_{j i}^{0}$, goes through a node $w_{k}, k \in \mathcal{D}_{j}$

(c) every loop $w_{j}$ to $w_{j}$ goes through a node $w_{k}, k \in \mathcal{D}_{j}$. $\square$

The proof is in Appendix X-E. The formulated conditions are used to make appropriate selections for the node variables that are to be measured and to be used as predictor inputs. In the following section it is shown that it is possible to identify the dynamics of the immersed network.

\section{Estimated Dynamics in Predictor Model}

In this section it is shown that the estimated dynamics between the predictor inputs and the module output $w_{j}$, are equal to $\breve{G}_{j k}^{0}\left(\mathcal{D}_{j}\right)$. The result confirms that the estimated dynamics are a consequence of the interconnection structure and the chosen predictor inputs. In addition conditions are presented that ensure that the estimates of $\breve{G}_{j k}^{0}\left(\mathcal{D}_{j}\right)$ are consistent. The results in this section are not specific to a particular identification method.

To concisely present the result, it is convenient to have a notation for a predictor which is a generalization of both the Direct and Two Stage Methods. Consider the predictor

$$
\begin{aligned}
& \hat{w}_{j}(t \mid t-1, \theta)=H_{j}^{-1}(q, \theta)\left(\sum_{k \in \mathcal{D}_{j}} G_{j k}(q, \theta) w_{k}^{(\mathcal{X})}(t)\right. \\
& \left.+\sum_{k \in \mathcal{P}_{j}} F_{j k}(q, \theta) r_{k}(t)\right)+\left(1-H_{j}^{-1}(q, \theta)\right) w_{j}(t)
\end{aligned}
$$

where $\mathcal{X}$ denotes a (sub)set of the variables $r_{k}, v_{k}, k \in$ $\{1, \ldots, L\}$. Note that both predictors (4) and (9) are special cases of the predictor (14). For the Direct Method, choose $\mathcal{X}=\left\{r_{k_{1}}, \ldots, r_{k_{n}}, v_{\ell_{1}}, \ldots, v_{\ell_{n}}\right\}$, where $\left\{k_{1}, \ldots, k_{n}\right\}=\mathcal{R}$, and $\left\{\ell_{1}, \ldots, \ell_{n}\right\}=\mathcal{V}$. Then $w_{k}^{(\mathcal{X})}=w_{k}$. For the Two Stage Method, choose $\mathcal{X}=\left\{r_{k_{1}}, \ldots, r_{k_{n}}\right\}$, where $\left\{k_{1}, \ldots, k_{n}\right\}=$ $\mathcal{T}_{j}$.

A key concept in the analysis of this section is the optimal output error residual, which will be discussed next. From (10), $w_{j}$ can be expressed in terms of $w_{k}, k \in \mathcal{D}_{j}$ as

$$
w_{j}=\sum_{k \in \mathcal{D}_{j}} \breve{G}_{j k}^{0} w_{k}+\sum_{k \in \mathcal{Z}_{j} \cap \mathcal{F}_{j}} \breve{F}_{j k}^{0} r_{k}+\sum_{k \in \mathcal{Z}_{j} \cap \mathcal{V}_{j}} \breve{F}_{j k}^{0} v_{k}+v_{j}+r_{j} .
$$

Note that by Lemma 1 some $\breve{F}_{j k}^{0}\left(q, \mathcal{D}_{j}\right)$ may be zero depending on the interconnection structure. Let $w_{k}$ be expressed in terms of a component dependent on the variables in $\mathcal{X}$, and a component dependent on the remaining variables, denoted $w_{k}=w_{k}^{(\mathcal{X})}+w_{k}^{(\perp \mathcal{X})}$. In addition, split the sum involving the $r_{k}$-dependent terms according to whether $r_{k}$ is in $\mathcal{P}_{j}$ or not. Then, from (15):

$$
\begin{aligned}
w_{j}=\sum_{k \in \mathcal{D}_{j}} \breve{G}_{j k}^{0} w_{k}^{(\mathcal{X})}+\sum_{k \in \mathcal{D}_{j}} \breve{G}_{j k}^{0} w_{k}^{(\perp \mathcal{X})}+\sum_{k \in \mathcal{P}_{j}} \breve{F}_{j k}^{0} r_{k} \\
\quad+\sum_{k \in\left(\left(\mathcal{Z}_{j} \cup\{j\}\right) \cap \mathcal{R}_{j}\right) \backslash \mathcal{P}_{j}} \breve{F}_{j k}^{0} r_{k}+\sum_{k \in \mathcal{Z}_{j} \cap \mathcal{V}_{j}} \breve{F}_{j k}^{0} v_{k}+v_{j} .
\end{aligned}
$$

When choosing an Output Error predictor (i.e. $H_{j}(q, \theta)=1$ ), with predictor inputs $w_{k}^{(\mathcal{X})}, k \in \mathcal{D}_{j}$ and $r_{k}, k \in \mathcal{P}_{j}$, the part of (16) that is not modeled can be lumped together into one term. This term is the optimal output error residual of $w_{j}$, and is denoted $p_{j}$ :

$$
p_{j}\left(\mathcal{D}_{j}\right):=\sum_{k \in \mathcal{D}_{j}} \breve{G}_{j k}^{0} w_{k}^{(\perp \mathcal{X})}+\sum_{k \in\left(\left(\mathcal{Z}_{j} \cup\{j\}\right) \cap \mathcal{R}_{j}\right) \backslash \mathcal{P}_{j}} \breve{F}_{j k}^{0} r_{k}+\breve{v}_{j},
$$

where $\breve{v}_{j}$ is given by $\sum_{k \in \mathcal{Z}_{j} \cap \mathcal{V}_{j}} \breve{F}_{j k}^{0} v_{k}+v_{j}$ in accordance with (12). Consequently, $w_{j}$ equals:

$$
w_{j}=\sum_{k \in \mathcal{D}_{j}} \breve{G}_{j k}^{0} w_{k}^{(\mathcal{X})}+\sum_{k \in \mathcal{P}_{j}} \breve{F}_{j k}^{0} r_{k}+p_{j} .
$$


In a system identification setting, the optimal output error residual of $w_{j}$ acts as the effective "noise" affecting $w_{j}$ (this is clear from (18)). It also corresponds to the unmodeled component of $w_{j}$.

The following theorem is the main result of this section. It characterizes conditions that correlation between the optimal output error residual of $w_{j}$ and the predictor inputs must satisfy so that it is possible to obtain consistent estimates of the dynamics between the predictor inputs. Such conditions are common in the identification literature. In openloop identification for instance it is well known that if the innovation is uncorrelated to the input consistent estimates are possible [21]. Similarly, it is known ([21]) that for the Direct Method in closed-loop, if the output noise is whitened and the whitened noise is uncorrelated to the plant input then consistent estimates of the plant are possible. The result that follows is an analogue to that reasoning adapted to identification in networks.

Theorem 1: Consider a dynamic network as defined in Section II-A that satisfies Assumption 1. Consider model structures with independently parameterized noise and module models. For given sets $\mathcal{D}_{j}, \mathcal{P}_{j}$, and $\mathcal{X}$ construct the predictor (14). Suppose the power spectral density of $\left[\begin{array}{lllllll}w_{j} & w_{k_{1}}^{(\mathcal{X})} & \ldots & w_{k_{n}}^{(\mathcal{X})} & r_{\ell_{1}} & \ldots & r_{\ell_{m}}\end{array}\right]^{T}$ where $\left\{k_{1}, \ldots, k_{n}\right\}=$ $\mathcal{D}_{j},\left\{\ell_{1}, \ldots, \ell_{m}\right\}=\mathcal{P}_{j}$ is positive definite for a sufficiently large number of frequencies $\omega_{k} \in(-\pi, \pi]$. Consider the conditions:

(a) $\overline{\mathbb{E}}\left[H_{j}^{-1}(q, \eta) p_{j}\left(t, \mathcal{D}_{j}\right) \cdot \Delta G_{j k}\left(q, \theta, \mathcal{D}_{j}\right) w_{k}^{(\mathcal{X})}(t)\right]=0, \forall k \in \mathcal{D}_{j}$, (b) $\overline{\mathbb{E}}\left[H_{j}^{-1}(q, \eta) p_{j}\left(t, \mathcal{D}_{j}\right) \cdot \Delta F_{j k}\left(q, \theta, \mathcal{D}_{j}\right) r_{k}(t)\right]=0, \forall k \in \mathcal{P}_{j}$, where $\Delta G_{j k}\left(\theta, \mathcal{D}_{j}\right)=\breve{G}_{j k}^{0}\left(\mathcal{D}_{j}\right)-G_{j k}(\theta)$, and $\Delta F_{j k}\left(\theta, \mathcal{D}_{j}\right)=$ $\breve{F}_{j k}^{0}\left(\mathcal{D}_{j}\right)-F_{j k}(\theta)$.

Then $G_{j k}\left(q, \theta^{*}\right)=\breve{G}_{j k}^{0}\left(q, \mathcal{D}_{j}\right)$, where $\breve{G}_{j k}^{0}\left(q, \mathcal{D}_{j}\right)$ is defined in Proposition 3, if for all $\theta \in \Theta$ :

1. Conditions (a) and (b) hold for all $\eta$, or

2. The equations of Conditions (a) and (b) hold for $\eta^{*}$ only, where $\eta^{*}=\arg \min \overline{\mathbb{E}}\left[\left(H_{j}^{-1}(q, \eta) p_{j}\left(t, \mathcal{D}_{j}\right)\right)^{2}\right]$, and $H_{j}^{-1}\left(q, \eta^{*}\right) p_{j}\left(t, \mathcal{D}_{j}\right)$ is white noise.

The proof can be found in Appendix X-F. The theorem can be interpreted as follows. In Case 1, consistent estimates are possible if the predictor inputs are uncorrelated to the optimal output error residual of $w_{j}$. This is analogous to the open loop situation. In Case 2, consistent estimates are possible if the whitened version of the optimal output error residual of $w_{j}$ is uncorrelated to the predictor inputs. This is analogous to the closed-loop Direct Method reasoning.

The condition on the power spectral density of $\left[\begin{array}{lllllll}w_{j} & w_{k_{1}}^{(\mathcal{X})} & \ldots & w_{k_{n}}^{(\mathcal{X})} & r_{\ell_{1}} & \ldots & r_{\ell_{m}}\end{array}\right]^{T}$ is a condition on the informativity of the data [30] (i.e. the data must be persistently exciting of sufficiently high order).

The main point of Theorem 1 is twofold:

1. The estimated transfer functions $G_{j k}\left(q, \theta^{*}\right)$ are consequences of the choice of $\mathcal{D}_{j}$. In particular, they are estimates of the transfer functions $\breve{G}_{j k}^{0}\left(q, \mathcal{D}_{j}\right)$ specified by the immersed network.

2. To present general conditions under which consistent estimates (of $\breve{G}_{j k}^{0}\left(q, \mathcal{D}_{j}\right)$ are possible.
Theorem 1 points to a notion of identifiability. For a given set $\mathcal{D}_{j}$, a particular module $G_{j i}^{0}$ is identifiable if $\breve{G}_{j i}^{0}=G_{j i}^{0}$. Thus, if the conditions of Proposition 4 are satisfied for a given set $\mathcal{D}_{j}$, then $G_{j i}^{0}$ is identifiable.

In the next two sections it is shown how Theorem 1 applies to both the Direct and Two Stage Methods respectively.

\section{Predictor Input Selection - Direct Method}

In this section it is shown how to satisfy the conditions of Theorem 1 using the Direct Method.

When using the Direct Method for identification in dynamic networks, there are three main mechanisms that ensure consistent estimates of $G_{j i}^{0}$ [19], [17] (the same mechanisms are present in the closed-loop Direct Method [21], [24], [23]):

1. the noise $v_{j}$ affecting the output $w_{j}$ is uncorrelated to all other noise terms $v_{n}, n \in \mathcal{V}_{j}$

2. every loop that passes through $w_{j}$ in the data generating system contains at least one delay, and

3. there exists a $\theta$ such that $H_{j}^{-1}(\theta) v_{j}=\breve{e}_{j}$ is white noise.

In Proposition 2 of [17] it is shown that for the choice $\mathcal{D}_{j}=\mathcal{N}_{j}$ and $\mathcal{P}_{j}=\emptyset$, these conditions plus a condition on the informativity of the data are sufficient in order to obtain consistent estimates of a module $G_{j i}^{0}$ embedded in the network. In the setup considered in this paper an additional mechanism plays a role, namely the choice of predictor inputs.

The following proposition presents conditions on the immersed network that ensure that Case 2 of Theorem 1 holds. The conditions reflect the three mechanisms presented above.

Proposition 5: Consider a dynamic network as defined in (2) that satisfies Assumption 1. Consider the immersed network constructed by removing $w_{n}, n \in \mathcal{Z}_{j}$ from the original network. The situation of Case 2 of Theorem 1 holds for the immersed network if:

(a) $\breve{v}_{j}$ is uncorrelated to all $\breve{v}_{k}, k \in \mathcal{D}_{j}$.

(b) There is a delay in every loop $w_{j}$ to $w_{j}$ (in the immersed network).

(c) If $\breve{G}_{j k}^{0}$ has a delay, then $G_{j k}(\theta)$ is parameterized with a delay.

(d) $p_{j}$ is not a function of any $r_{n}, n \in \mathcal{R}$.

(e) There exists a $\eta$ such that $H_{j}^{-1}(q, \eta) p_{j}(t)$ is white noise. The proof can be found in Appendix X-G.

In the following subsections, the conditions of Proposition 5 are interpreted in terms of what they mean in the original network. In Subsection V-A it is shown what conditions can be imposed in the original network in order to ensure that $\breve{v}_{j}$ is uncorrelated to $\breve{v}_{k}, k \in \mathcal{D}_{j}$ (i.e Condition (a) of Proposition 5 holds).In Subsection V-B it is shown under which conditions $p_{j}$ is not a function of external variables (i.e. Condition (d) of Proposition 5 holds). In Subsection V-C a version of Proposition 5 is presented where all the conditions are stated only in terms of the original network.

\section{A. Correlation of Noise}

In this section conditions are presented that ensure that $\breve{v}_{j}$ is uncorrelated to $\breve{v}_{k}, k \in \mathcal{D}_{j}$. The conditions are presented using only variables in the original network. 
Recall from (12) that $\breve{v}_{k}$ is a filtered sum of $v_{n}, n \in \mathcal{Z}_{j} \cup\{k\}$,

$$
\breve{v}_{k}(t)=\sum_{n \in \mathcal{Z}_{j}} \breve{F}_{j n}^{0}\left(q, \mathcal{D}_{j}\right) v_{n}+\breve{F}_{j j}^{0}\left(q, \mathcal{D}_{j}\right) v_{j}(t) .
$$

Consider 2 variables $\breve{v}_{k_{1}}$ and $\breve{v}_{k_{2}}$. Suppose that there is a path from another variable $v_{n}, n \in \mathcal{Z}_{j}$ to both $w_{k_{1}}$ and $w_{k_{2}}$. By Lemma 1 both $\breve{F}_{k_{1} n}^{0}$ and $\breve{F}_{k_{2} n}^{0}$ are non-zero in this situation. Consequently, as can be see from (19) both $\breve{v}_{k_{1}}$ and $\breve{v}_{k_{2}}$ are functions of $v_{n}$, with the result that $\breve{v}_{k_{1}}$ and $\breve{v}_{k_{2}}$ are correlated. Thus, due to the presence of $v_{n}$ and the interconnection structure of the network, $\breve{v}_{k_{1}}$ and $\breve{v}_{k_{2}}$ are correlated. In this case $v_{n}$ is a confounding variable. In statistics, and in particular in statistical inference, a confounding variable is a variable that is not known (or measured) and causally affects both the output variable and the input variable [31]. The induced correlation between input and output is however not caused by a direct causal relation between the input and output. In the framework of this paper consider the following definition.

Definition 1: Consider a particular output variable $w_{j}$ and a set $\mathcal{D}_{j}$ of predictor inputs. In this modeling setup, a variable $v_{\ell}$ is a confounding variable if the following conditions hold:

(a) There is a path from $v_{\ell}$ to $w_{j}$ that passes only through $w_{m}, m \in \mathcal{Z}_{j}$.

(b) There is a path from $v_{\ell}$ to one or more $w_{k}, k \in \mathcal{D}_{j}$ that passes only through $w_{m}, m \in \mathcal{Z}_{j}$.

The following is an example of a confounding variable.

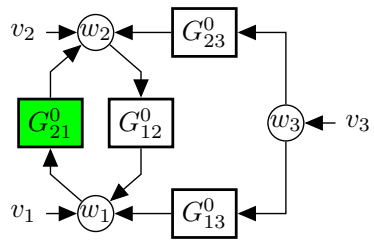

Fig. 6. Network that is analyzed in Example 5.

Example 5: Consider the network shown in Fig. 6. Suppose that the objective is to obtain a consistent estimate of $G_{21}^{0}$ (denoted in green) using the Direct Method. Let $j=2$, and choose $\mathcal{D}_{2}=\{1\}$. By Definition $1, v_{3}$ is a confounding variable. The expressions for $\breve{v}_{1}$ and $\breve{v}_{2}$ for this network are:

$$
\breve{v}_{1}=v_{1}+G_{13}^{0} v_{3} \quad \text { and } \quad \breve{v}_{2}=v_{2}+G_{23}^{0} v_{3} .
$$

Clearly, the confounding variable $v_{3}$ induces a correlation between $\breve{v}_{1}$ and $\breve{v}_{2}$.

The presence of confounding variables is not the only way that $\breve{v}_{k_{1}}$ and $\breve{v}_{k_{2}}$ could become correlated. Suppose that $\breve{v}_{k_{1}}$ is a function of $v_{n}$, and $\breve{v}_{k_{2}}$ is a function of $v_{m}$. If $v_{n}$ and $v_{m}$ are correlated, then $\breve{v}_{k_{1}}$ and $\breve{v}_{k_{2}}$ are correlated.

The following proposition presents conditions that ensure $\breve{v}_{j}$ is uncorrelated to all $\breve{v}_{k}, k \in \mathcal{D}_{j}$.

Proposition 6: Consider a dynamic network as defined in (2) that satisfies Assumption 1. Consider the immersed network constructed from the internal variables, $\left\{w_{k}\right\}, k \in \mathcal{D}_{j}$. The disturbance term $\breve{v}_{j}$ (as defined in (12)) is uncorrelated to all $\breve{v}_{k}, k \in \mathcal{D}_{j}$ if the following conditions hold:

(a) $v_{j}$ is uncorrelated to all $v_{k}, k \in \mathcal{D}_{j}$ and to all variables $v_{n}, n \in \mathcal{Z}_{j}$ that have paths to any $w_{k}, k \in \mathcal{D}_{j}$ that pass only through nodes $w_{\ell}, \ell \in \mathcal{Z}_{j}$. (b) All $v_{k}, k \in \mathcal{D}_{j}$ are uncorrelated to all $v_{n}, n \in \mathcal{Z}_{j}$ that have a path to $w_{j}$ that passes only through nodes in $\mathcal{Z}_{j}$.

(c) All $v_{n}, n \in \mathcal{Z}_{j}$ are uncorrelated to each other

(d) No variable $v_{k}, k \in \mathcal{Z}_{j}$ is a confounding variable.

The proof can be found in Appendix X-H.

Remark 3: Suppose that all $v_{k}, k \in \mathcal{V}$ are uncorrelated. Then Conditions (a) - (c) hold for any $\mathcal{D}_{j}$. However, whether Condition (d) holds depends on the interconnection structure and the choice of $\mathcal{D}_{j}$.

\section{B. Adding External Excitation}

External variables are not strictly necessary to ensure that the data is informative when using the direct method as long as the noise that is driving the system is sufficiently exciting. However, external excitation can be beneficial in order to reduce the variance of the estimates, or provide extra excitation in a frequency range of interest.

Whenever there is an external variable $r_{k}$ acting as a "disturbance" on the output variable $w_{j}$ (i.e. $p_{j}$ contains an element which is due to the external variable $r_{k}$ ), it makes sense to model that component. This happens whenever there is a path $r_{k}$ to $w_{j}$ that passes only through $w_{k}, k \in \mathcal{Z}_{j}$. Thus, in this case, choose the set $\mathcal{P}_{j}=\{k\}$ so that $r_{k}$ is included as a predictor input (i.e. the dynamics from $r_{k}$ to $w_{j}$ are modeled). The advantage of this scheme is that the power of the optimal output error residual is reduced by eliminating known variables from $p_{j}$, i.e. the signal to noise ratio is increased. Consequently, $p_{j}$ is only a function of $v$ 's (Condition (d) of Proposition 5 holds).

\section{Main Result - Direct Method}

Conditions are presented so that the Direct Method will result in consistent estimates of $\breve{G}_{j i}^{0}\left(\mathcal{D}_{j}\right)$. In Proposition 5 the conditions were stated in terms of the immersed network. In the following proposition the conditions are stated in terms of the original network.

Proposition 7: Consider a dynamic network as defined in (2) that satisfies Assumption 1. Let $\left\{w_{k}\right\}, k \in \mathcal{D}_{j}$ and $\left\{r_{k}\right\}$, $k \in \mathcal{P}_{j}$ be the set of internal and external variables respectively that are included as inputs to the predictor (4). The set $\mathcal{P}_{j}$ is constructed to satisfy the condition that $k \in \mathcal{P}_{j}$ if and only if there exists a path from $r_{k}$ to $w_{j}$, that passes only through nodes in $\mathcal{Z}_{j}$. Consistent estimates of $\breve{G}_{j i}^{0}$ are obtained using the Direct Method formulated in Algorithm 1 if the following conditions are satisfied:

(a) There is a delay in every loop $w_{j}$ to $w_{j}$.

(b) $v$ satisfies the conditions of Proposition 6.

(c) The power spectral density of $\left[\begin{array}{llllll}w_{j} & w_{k_{1}} & \cdots & w_{k_{\mathfrak{n}}} r_{\ell_{1}} & \cdots & r_{\ell_{m}}\end{array}\right]^{T}, k_{*} \in \mathcal{D}_{j}, \ell_{*} \in \mathcal{P}_{j}$ is positive definite for a sufficiently large number of frequencies $\omega_{k} \in(-\pi, \pi]$.

(d) The parameterization is chosen flexible enough, i.e. there exist parameters $\theta$ and $\eta$ such that $G_{j k}(q, \theta)=$ $\breve{G}_{j k}^{0}\left(q, \mathcal{D}_{j}\right), \forall k \in \mathcal{D}_{j}, F_{j k}(q, \theta)=\breve{F}_{j k}^{0}\left(q, \mathcal{D}_{j}\right), \forall k \in \mathcal{P}_{j}$, and $H_{j}(q, \eta)=\breve{H}_{j}^{0}\left(q, \mathcal{D}_{j}\right)$.

(e) If $\breve{G}_{j k}^{0}$ has a delay, then $G_{j k}(\theta)$ is parameterized with a delay. 
Proof: The proof follows almost directly from Theorem 1 and Propositions 5 and 6. It remains to be shown that $p_{j}=\breve{v}_{j}$ (i.e. Condition (d) of Proposition 5 holds).

By Lemma $1 \breve{F}_{j k}^{0}, k \in \mathcal{D}_{j}$ is zero unless there is a path from $r_{k}$ to $w_{j}$ which passes only through $w_{n}, n \in \mathcal{Z}_{j}$. From (17) and by the way $\mathcal{P}_{j}$ is constructed it follows that there are no $r$ terms present in $p_{j}$. Consequently, $p_{j}=\breve{v}_{j}$.

Remark 4: In Proposition 7 conditions have been presented which, if satisfied, ensure that consistent estimates of $\breve{G}_{j k}^{0}\left(q, \mathcal{D}_{j}\right), k \in \mathcal{D}_{j}$ as defined by the immersed network are obtained. If the set $\mathcal{D}_{j}$ is chosen such that $\breve{G}_{j i}^{0}\left(q, \mathcal{D}_{j}\right)=$ $G_{j i}^{0}(q)$ (i.e. the $\mathcal{D}_{j}$ is chosen such that the conditions of Proposition 4 are satisfied) then Proposition 7 shows under which conditions $G_{j i}^{0}$ can be consistently identified.

The reason that Condition (a) and exact noise modeling are required is due to the presence of a (feedback) path from $w_{j}$ to at least one $w_{k}, k \in \mathcal{D}_{j}$. If there is no such feedback, then the conditions of Proposition 7 simplify considerably. Similarly, since, it is the variable $v_{j}$ that is causing the problems when there is such a feedback path, if it is not present, the conditions can be simplified.

Corollary 1: Consider the situation of Proposition 7. If there is no path from $w_{j}$ to any $w_{k}, k \in \mathcal{D}_{j}$, or if $v_{j}$ is not present in the network, then Conditions (a) and (e) can be omitted, and Condition (d) can be changed to:

(d') The parameterization is chosen flexible enough, i.e. there exists a parameter $\theta$ such that $G_{j k}(q, \theta)=\breve{G}_{j k}^{0}\left(q, \mathcal{D}_{j}\right)$, $\forall k \in \mathcal{D}_{j}, F_{j k}(q, \theta)=\breve{F}_{j k}^{0}\left(q, \mathcal{D}_{j}\right), \forall k \in \mathcal{P}_{j}$.

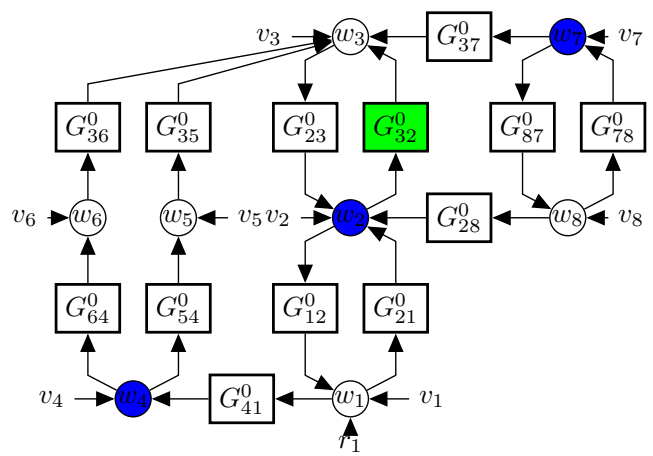

Fig. 7. Network that is analyzed in Examples 6 and 8.

Example 6: Consider the dynamic network shown in Fig. 7. Suppose the objective is to obtain consistent estimates of $G_{32}^{0}$ (denoted in green) using the Direct Method.

First, we show how to choose the set $\mathcal{D}_{3}$ such that $\breve{G}_{32}^{0}\left(q, \mathcal{D}_{j}\right)$ in the immersed network is equal to $G_{32}^{0}(q)$ (i.e. $\mathcal{D}_{j}$ is chosen such that it satisfies the conditions of Proposition 4). Besides $G_{32}^{0}$ there are several paths from $w_{2}$ to $w_{3}$ :

$$
\begin{aligned}
& w_{2} \rightarrow w_{1} \rightarrow w_{4} \rightarrow w_{5} \rightarrow w_{3}, \\
& w_{2} \rightarrow w_{1} \rightarrow w_{4} \rightarrow w_{6} \rightarrow w_{3}
\end{aligned}
$$

for instance. All paths from $w_{2}$ to $w_{3}$ (not including $G_{32}^{0}$ ) pass through either the nodes $w_{1}$ and $w_{2}$, the nodes $w_{4}$ and $w_{2}$. Thus, Condition (b) of Proposition 4 is satisfied for $\mathcal{D}_{3}=$ $\{1,2\}$ and $\mathcal{D}_{3}=\{2,4\}$.

Since all loops from $w_{3}$ pass through $w_{2}$, Condition (c) of Proposition 4 is also satisfied for both these choices of $\mathcal{D}_{3}$.
For both of these choices, $v_{7}$ and $v_{8}$ are confounding variables (Condition (b) of Proposition 7 is not satisfied). However, if $w_{7}$ is included as a predictor input, then there are no more confounding variables.

By this reasoning two possible choices for $\mathcal{D}_{3}$ that lead to consistent estimates of $G_{32}^{0}$ are $\{2,4,7\}$ (denoted in blue) and $\{2,1,7\}$. In either case, $\mathcal{P}_{3}$ should be chosen as $\emptyset$.

Another possible choice for $\mathcal{D}_{3}=\{2,5,6,7\}=\mathcal{N}_{3}$. It is interesting that the previous sets $\mathcal{D}_{3}$ are strictly smaller than $\mathcal{N}_{3}$, and are not even subsets of $\mathcal{N}_{3}$.

The choice $\mathcal{D}_{j}=\mathcal{N}_{j}, \mathcal{P}_{j}=\emptyset$ always satisfies the Conditions of Proposition 4 and confounding variables are never present. This is the choice that is made in [17].

In the following section an analogue to Proposition 7 is presented for the Two-Stage Method.

\section{Vi. Predictor Input Selection - Two Stage Method}

A guiding principle to ensure consistent estimates that has been presented in Theorem 1 is that the optimal output error residual of $w_{j}$ should be uncorrelated to the predictor inputs. For the Two Stage Method this condition is enforced by projecting the predictor inputs onto the external variables. Consequently, the predictor inputs are only functions of $r_{m}$, $m \in \mathcal{T}_{j}$. As long as the unmodeled component of $w_{j}$ is not a function of $r_{m}, m \in \mathcal{T}_{j}$ then Conditions (a) and (b) of Theorem 1 are satisfied.

Proposition 8: Consider a dynamic network as defined in (2) that satisfies Assumption 1. Let $\left\{r_{m}\right\}, m \in \mathcal{T}_{j}$ be the external input(s) onto which will be projected. Let $\left\{w_{k}^{\left(\mathcal{T}_{j}\right)}\right\}$, $k \in \mathcal{D}_{j}$ and $\left\{r_{k}\right\}, k \in \mathcal{P}_{j}$ be the sets of (projections of) internal and external variables respectively that are included as inputs to the predictor (9). The set $\mathcal{P}_{j}$ is constructed to satisfy the condition that $k \in \mathcal{P}_{j}$ if and only if there exists a path from $r_{k}$ to $w_{j}, k \in \mathcal{T}_{j}$, that passes only through nodes in $\mathcal{Z}_{j}$. Consistent estimates of $G_{j i}^{0}$ are obtained using the Two Stage Method (Algorithm 2) if the following conditions hold:

(a) Every $r_{k}, k \in \mathcal{T}_{j}$ is uncorrelated to all $r_{m}, m \notin \mathcal{T}_{j}$, except those $r_{m}$ for which there is no path to $w_{j}$.

(b) The power spectral density of $\left[w_{k_{1}}^{\left(\mathcal{T}_{j}\right)} \cdots w_{k_{\mathfrak{n}}}^{\left(\mathcal{T}_{j}\right)} r_{m_{1}} \cdots r_{m_{n}}\right]^{T}$, $k_{*} \in \mathcal{D}_{j}, m_{*} \in \mathcal{P}_{j}$, is positive definite for a sufficient number of frequencies $\omega_{k} \in(-\pi, \pi]$

(c) The parameterization is chosen flexible enough, i.e. there exists a parameter $\theta$ such that $G_{j k}(q, \theta)=\breve{G}_{j k}^{0}\left(q, \mathcal{D}_{j}\right)$, $\forall k \in \mathcal{D}_{j}, F_{j k}(q, \theta)=\breve{F}_{j k}^{0}\left(q, \mathcal{D}_{j}\right), \forall k \in \mathcal{P}_{j}$

For a proof, see Appendix X-I.

Note that in order for Condition (b) to hold, there must be a path from at least one $r_{m}, m \in \mathcal{T}_{j}$ to $w_{i}$. If not, then $w_{i}^{\left(\mathcal{T}_{j}\right)}=0$ and the power spectral density of Condition (b) will not be positive definite.

Remark 5: The condition on the order of excitation of the data (Condition (b)) can be satisfied if there is one external variable present for each predictor input. This is however just a sufficient condition. For more information on how the network dynamics add excitation to the data so that fewer external variables are required see [32] for instance.

Remark 6: In the discussion thus far, we have not allowed the choice of $w_{j}$ as a predictor input (by Condition (a) in 
Proposition $4, j$ is not allowed to be in $\mathcal{D}_{j}$ ). It can be shown that $w_{j}$ can be used as a predictor input to consistently identify $G_{j i}^{0}$ using the Two-Stage method if $r_{j}$ is present (and Conditions (a) - (c) of Proposition 8 are satisfied). Moreover, it can also be shown that if $r_{j}$ is not present, then it is not possible to choose $w_{j}$ as a predictor input to consistently identify $G_{j i}^{0}$ using the Two-Stage Method (as proved in [33]). The advantage of choosing $w_{j}$ as a predictor input is that Condition (c) of Proposition 4 is automatically satisfied without including any other variables.

Remark 7: The Conditions presented in Proposition 8 do not change if there is measurement noise present on the measurements of $w_{k}, k \in \mathcal{D}_{j}$. The Two Stage method still results in consistent estimates of $\breve{G}_{j i}^{0}$ in the presence of measurement noise, as long as the $r$ 's are exactly known. This observation is further explored and generalized in [34].

Compare the conditions of the Direct and Two Stage Methods. For the Two Stage Method there are no restrictions on algebraic loops, the correlation of the noise terms, or the presence of confounding variables. However, to use the Two Stage Method at least one external variable $r_{m}$ must be present that affects $w_{i}$ (this is not the case for the Direct Method). Moreover, the excitation conditions of the Two Stage Method are stricter than those of the Direct Method.

From the perspective of reducing the variance of an estimate, it is desirable to project onto as many external variables as possible, since this increases the power of the predictor inputs relative to the optimal output error residual (not projecting onto a particular external variable means that the power of the predictor inputs is less, and that particular external variable becomes part of the unmodeled component of the output, increasing the power of the optimal output error residual).

Example 7: Recall the network of Example 3 shown in Fig. 4. Suppose that the objective is to obtain an estimate of $G_{21}^{0}$ (denoted in green) using the Two Stage Method. Choose an output error model structure $\left(H_{2}(q, \theta)=1\right)$. Choose $\mathcal{D}_{2}=$ $\{1,3,4\}$. For this choice of $\mathcal{D}_{2}$ all conditions of Proposition 4 are satisfied, and therefore $\breve{G}_{21}^{0}=G_{21}^{0}$. To ensure that the estimate of $\breve{G}_{21}^{0}$ is consistent, $\mathcal{P}_{2}$ must also be chosen properly.

Choose to project the predictor inputs onto $r_{1}$ and $r_{5}\left(\mathcal{T}_{2}=\right.$ $\{1,5\})$. Thus, by Proposition $8 \mathcal{P}_{2}$ is set to $\{5\}$, since there is a path from $r_{5}$ to $w_{2}$ that passes only through $w_{n} n \in \mathcal{Z}_{2}=\{5\}$.

Now consider projecting only onto $r_{1}$. In this case, by Proposition $8, \mathcal{P}_{2}$ is set to $\emptyset$.

Finally, consider the choice $\mathcal{D}_{2}=\{1,2,5\}$. Futhermore, choose to project onto both $r_{1}$ and $r_{5}$. In this case, by Proposition $8, \mathcal{P}_{2}$ is set to $\emptyset$. In this case, due to the different choice of $\mathcal{D}_{2}, \mathcal{P}_{2}$ can be chosen as $\emptyset$ even though $\mathcal{T}_{2}=\{1,5\}$ just like in the first case considered in this example.

Example 8: Consider the same network as in Example 6, shown in Fig. 7. Suppose the objective is to obtain consistent estimates of $G_{32}^{0}$ (marked in green) using the Two Stage Method. Choose $r_{1}$ as the external variable to project onto $\left(\mathcal{T}_{3}=\{1\}\right)$. By the same reasoning as in Example 6, choosing $\mathcal{D}_{3}=\{1,2\}$ or $\{2,4\}$ satisfies the conditions of Proposition 4. However, in this case (unlike for the Direct Method) both these choices of $\mathcal{D}_{3}$ satisfy all the remaining conditions of Proposition 8 (since confounding variables are not an issue for the Two Stage Method).

Finally, $\mathcal{P}_{3}$ must be chosen as stated in Proposition 8. There are two independent paths from $r_{1}$ to $w_{3}$,

$$
r_{1} \rightarrow w_{4} \rightarrow w_{6} \rightarrow w_{3} \quad \text { and } \quad r_{1} \rightarrow w_{2} \rightarrow w_{3}
$$

both of which pass through a variable $w_{n}, n \in \mathcal{D}_{3}$, so $\mathcal{P}_{3}$ should be chosen as $\emptyset$.

\section{Algorithmic Aspects}

In this section an algorithm is presented that provides a way to check the conditions that the set $\mathcal{D}_{j}$ must satisfy in order to ensure that $\breve{G}_{j i}^{0}\left(q, \mathcal{D}_{j}\right)$ of the immersed network is equal to $G_{j i}^{0}(q)$ of the original network (see Proposition 4). The algorithm uses tools from graph theory, therefore, before presenting the result, consider the following definitions.

Definition 2 ( $A-B$ path [35]): Given a directed graph $\mathbb{G}$ and sets of nodes $A$ and $B$. Denote the nodes in the graph by $x_{i}$. A path $\mathbb{P}=x_{0} x_{1} \cdots x_{k}$, where the $x_{i}$ are all distinct, is an $A-B$ path if $V(\mathbb{P}) \cap A=\left\{x_{0}\right\}$, and $V(\mathbb{P}) \cap B=\left\{x_{k}\right\}$.

Definition 3 ( $A$ - $B$ Separating Set [35]): Given a directed graph $\mathbb{G}$, and sets of nodes $A, B \subset V(\mathbb{G})$, a set $X \subseteq V(\mathbb{G})$ is an $A$ - $B$ separating set if the removal of the nodes in $X$ results in a graph with no $A-B$ paths.

The following notation will be useful in order to reformulate the conditions of Proposition 4 using the notion of separating sets. Let the node $w_{j}$ be split into two nodes, $w_{j}^{+}$to which all incoming edges (of $w_{j}$ ) are connected and $w_{j}^{-}$to which all outgoing edges (of $w_{j}$ ) are connected. The new node $w_{j}^{+}$is connected to $w_{j}^{-}$with the edge $G_{j^{+} j^{-}}=1$. Let $w_{i}^{+}$and $w_{i}^{-}$ be defined analogously.

Proposition 9: The conditions of Proposition 4 can be reformulated as: the set $\mathcal{D}_{j}$ is a $\left\{w_{i}^{+}, w_{j}^{-}\right\}-\left\{w_{j}^{+}\right\}$separating set.

Proof: The conditions of Proposition 4 can be rewritten as follows. The set $\mathcal{D}_{j}$ satisfies the following conditions:

1. $\mathcal{D}_{j} \backslash\{i\}$ is a $\left\{w_{i}\right\}-\left\{w_{j}\right\}$ separating set for the network with path $G_{j i}^{0}$ removed,

2. $\mathcal{D}_{j}$ is a $\left\{w_{j}^{-}\right\}-\left\{w_{j}^{+}\right\}$separating set.

These two conditions can be formulated as the single condition of the proposition.

Note that $w_{i}$ must always chosen to be in $\mathcal{D}_{j}$ to ensure that $\mathcal{D}_{j}$ is a $\left\{w_{i}^{+}, w_{j}^{-}\right\}-\left\{w_{j}^{+}\right\}$separating set (i.e. Condition (a) of Proposition 4 is automatically satisfied). This is because there is always a path $w_{i}^{+} \rightarrow w_{i}^{-} \rightarrow w_{2}^{+}$. Consequently, $w_{i}^{-}$must be chosen in the set $\mathcal{D}_{j}$.

The advantage of reformulating the conditions in terms of separating sets is that there exist tools from graph theory to check if a given set is a separating set or to find (the smallest possible) separating sets [35], [36].

Example 9: Consider the network shown in Fig. 8. Suppose that the objective is to obtain consistent estimates of $G_{21}^{0}$ (denoted in green). Both $w_{1}$ and $w_{2}$ have been split into two nodes as described above.

By Proposition 9 the conditions of Proposition 4 are satisfied for the given network if $\mathcal{D}_{2}$ is a $\left\{w_{1}^{+}, w_{2}^{-}\right\}-\left\{w_{2}^{+}\right\}$separating set. The outgoing set $\left\{w_{1}^{+}, w_{2}^{-}\right\}$is denoted in brown, and the incoming set $\left\{w_{2}^{+}\right\}$is denoted in orange in the figure. 


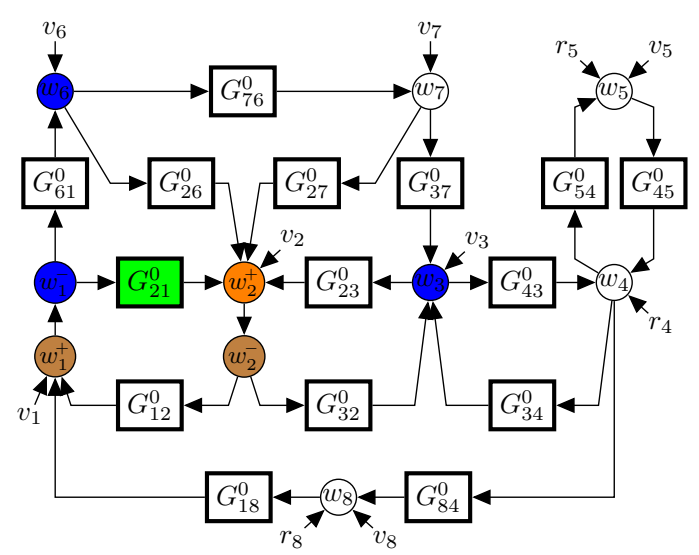

Fig. 8. Example of an interconnected network used in Example 9.

There are many possible choices of $\mathcal{D}_{2}$, but the smallest choice, $\left\{w_{1}^{-}, w_{6}, w_{3}\right\}$, is denoted in blue. It is easy to verify that all paths from the brown set to the orange set pass through a node in the blue set.

\section{DISCUSSION}

The approach presented in this paper is a local approach in the sense that only a (small) subset of internal variables are required to identify a particular module embedded in the network. Therefore, even for large networks, the numerical complexity of obtaining an estimate of a particular module can be limited by proper choice of predictor inputs. If the number of predictor inputs is large it may be attractive to rely on linear regression schemes such as ARX, FIR [21] and orthogonal basis function expansions [37], as well as IV-type and subspace algorithms [21].

While we have restricted this paper to dealing with questions of consistency, variance properties of estimates will be highly relevant to consider as a function of measured node signals as predictor inputs, as well as of external variables present.

\section{CONCLUSION}

In this paper, identification in dynamics networks has been investigated. In a dynamic network, unlike in open or closed loop systems, there are many options as to which variables to include as predictor inputs. It has been shown that when identifying in networks, the obtained estimates are consequences of the (chosen) set of predictor inputs. In particular, the obtained estimates are estimates of the dynamics defined by the immersed network. Conditions on the predictor inputs have been presented such that it is possible to obtain consistent estimates of a module embedded in a dynamic network using either the Direct or Two Stage methods of identification. These conditions are useful since they enable the user to design a least expensive sensor placement scheme or check if it is possible to avoid using particular variables in the identification experiment for instance.

\section{Appendix}

\section{A. Proof of Proposition 1}

Rather than checking the conditions of Theorem 2 in [25] it is more straightforward to provide a direct proof of the result.
The following Lemma is used in proving Proposition 1. The proof can be found in [33].

Lemma 2: Let $\mathcal{G}$ be a $n \times m$ matrix of transfer functions, with $n \leq m$. Suppose all principal minors of $\mathcal{G}$ are non-zero. The matrix $\mathcal{G}$ can be uniquely factored as $(I-G)^{-1} F$, where $G$ and $F$ have the structure defined in (11).

Now follows the proof of Proposition 1.

Proof: Any network can be expressed as

$$
\left[\begin{array}{l}
w_{j}(t) \\
w_{\mathcal{D}}(t)
\end{array}\right]=\mathcal{G}^{0}(q)\left[\begin{array}{l}
r_{j}(t)+v_{j}(t) \\
r_{\mathcal{D}}(t)+v_{\mathcal{D}}(t) \\
r_{\mathcal{Z}}(t)+v_{\mathcal{Z}}(t)
\end{array}\right] .
$$

Because the network is well posed, the principal minors of $\mathcal{G}$ are all non-zero. Thus, by Lemma $2, \mathcal{G}$ can be uniquely factored into $\breve{G}^{0}$ and $\breve{F}^{0}$ with the structure (11). If there is an index $\ell$ such that both $v_{\ell}$ and $r_{\ell}$ are not present, then setting the corresponding column of $\breve{F}^{0}$ to zero has no effect on in the validity of (10) with respect to the signals.

\section{B. Proof of Proposition 2}

Proof: The proof proceeds by showing that Algorithm 3 results in matrices $\breve{G}^{0}$ and $\breve{F}^{0}$ of the form in Proposition 1.

In Step 2c of Algorithm 3 no path starting from $v_{k}$ (or $r_{k}$ ), $k \in \mathcal{D}_{j}$ is ever lifted. Moreover, in the framework considered in this paper, in the original network, $v_{k}, k \in \mathcal{V}$ (or $r_{k}, k \in \mathcal{R}$ ) only has a path to $w_{k}$. It follows that in the immersed network, $v_{k}$ (or $r_{k}$ ), $k \in \mathcal{D}_{j}$ only has a path to $w_{k}$. Thus, all the offdiagonal entries of the leading square matrix of $\breve{F}^{i^{0}}$ are zero, which shows that the form of $\breve{F}^{i}$ is the same as that of $\breve{F}^{0}$.

In Step 3 of the algorithm all self-loops are removed. Thus the diagonal entries of $\breve{G}^{i}$ are set to zero. This shows that $\breve{G}^{i^{0}}$ and $\breve{G}^{0}$ have the same form.

By the uniqueness result of Proposition 1 it follows that $\breve{F}^{i^{0}}=\breve{F}^{0}$ and $\breve{G}^{0}=\breve{G}^{i^{0}}$

\section{Proof of Proposition 3}

Proof: The proof proceeds by starting with the original network (2) and removing the internal variables $w_{k}, k \in \mathcal{Z}_{j}$ from the equations. The proofs proceeds at a signal level. At the end of the proof, matrices $\breve{G}^{0}$ and $\breve{F}^{0}$ are obtained of the form required by Proposition 1. Consequently, uniqueness of the matrices is ensured.

Given a network of the form (3), the variables $w_{\mathcal{z}}$ must be removed from the equation. This is done by expressing $w_{z}$ in terms of $w_{k}, k \in\{j\} \cup \mathcal{D}_{j}, v_{k}, k \in \mathcal{Z}_{j}$, and $r_{k}, k \in \mathcal{Z}_{j}$ :

$$
\begin{aligned}
w_{\mathcal{Z}} & =G_{\mathcal{Z} j}^{0} w_{j}+G_{\mathcal{Z D}}^{0} w_{\mathcal{D}}+G_{\mathcal{Z Z}} w_{\mathcal{Z}}+v_{\mathcal{Z}}+r_{\mathcal{Z}} \\
& =\left(I-G_{\mathcal{Z Z}}\right)^{-1}\left(G_{\mathcal{Z} j} w_{j}+G_{\mathcal{Z D}} w_{\mathcal{D}}+v_{\mathcal{Z}}+r_{\mathcal{Z}}\right) .
\end{aligned}
$$

where the inverse exists by Assumption 1. In order to eliminate $w_{\mathcal{Z}}$ from the expression of $\left[w_{j} w_{\mathcal{D}}\right]$, first express $\left[w_{j} w_{\mathcal{D}}\right]$ in terms of $w_{\mathcal{Z}}$, and then substitute in (20):

$$
\begin{aligned}
{\left[\begin{array}{c}
w_{j} \\
w_{\mathcal{D}}
\end{array}\right]=} & {\left[\begin{array}{cc}
0 & G_{j \mathcal{D}} \\
G_{\mathcal{D} j} & G_{\mathcal{D D}}
\end{array}\right]\left[\begin{array}{l}
w_{j} \\
w_{\mathcal{D}}
\end{array}\right]+\left[\begin{array}{l}
G_{j \mathcal{Z}} \\
G_{\mathcal{D Z}}
\end{array}\right] w_{\mathcal{Z}}+\left[\begin{array}{l}
v_{j} \\
v_{\mathcal{D}}
\end{array}\right]+\left[\begin{array}{c}
r_{j} \\
r_{\mathcal{D}}
\end{array}\right] } \\
= & {\left[\begin{array}{cc}
0 & G_{j \mathcal{D}} \\
G_{\mathcal{D} j} & G_{\mathcal{D D}}
\end{array}\right]\left[\begin{array}{l}
w_{j} \\
w_{\mathcal{D}}
\end{array}\right]+\left[\begin{array}{l}
G_{j \mathcal{Z}} \\
G_{\mathcal{D Z}}
\end{array}\right]\left(I-G_{\mathcal{Z Z}}\right)^{-1}\left[G_{\mathcal{Z} j} G_{\mathcal{Z D}}\right]\left[\begin{array}{l}
w_{j} \\
w_{\mathcal{D}}
\end{array}\right] } \\
& +\left[\begin{array}{l}
G_{j \mathcal{z}} \\
G_{\mathcal{D Z}}
\end{array}\right]\left(I-G_{\mathcal{Z Z}}\right)^{-1}\left(r_{\mathcal{Z}}+v_{\mathcal{Z}}\right)+\left[\begin{array}{l}
v_{j} \\
v_{\mathcal{D}}
\end{array}\right]+\left[\begin{array}{l}
r_{j} \\
r_{\mathcal{D}}
\end{array}\right] .
\end{aligned}
$$


Collect all the $v$ 's and $r$ 's into a single vector:

$$
\begin{aligned}
{\left[\begin{array}{c}
w_{j} \\
w_{\mathcal{D}}
\end{array}\right]=} & \left(\left[\begin{array}{cc}
0 & G_{j \mathcal{D}} \\
G_{\mathcal{D} j} & G_{\mathcal{D D}}
\end{array}\right]+\left[\begin{array}{l}
G_{j \mathcal{Z}} \\
G_{\mathcal{D Z}}
\end{array}\right]\left(I-G_{\mathcal{Z Z}}\right)^{-1}\left[G_{\mathcal{Z} j} G_{\mathcal{Z D}}\right]\right)\left[\begin{array}{l}
w_{j} \\
w_{\mathcal{D}}
\end{array}\right] \\
& +\left[\begin{array}{lll}
1 & 0 & G_{j \mathcal{Z}}\left(I-G_{\mathcal{Z Z}}\right)^{-1} \\
0 & I & G_{\mathcal{D Z}}\left(I-G_{\mathcal{Z Z}}\right)^{-1}
\end{array}\right]\left[\begin{array}{l}
r_{j}+v_{j} \\
r_{\mathcal{D}}+v_{\mathcal{D}} \\
r_{\mathcal{Z}}+v_{\mathcal{Z}}
\end{array}\right] .
\end{aligned}
$$

From the statement of the Proposition, the matrix preceding $\left[w_{j} w_{\mathcal{D}}\right]^{T}$ is $\tilde{G}^{0}$, and the matrix preceding the $r$ and $v$ terms is $\tilde{F}^{0}$. To put the matrices $\tilde{G}^{0}$ and $\tilde{F}^{0}$ into the form required by Proposition 1 , the diagonals of $\tilde{G}^{0}$ must be removed. Let $D$ denote the diagonal entries of $\tilde{G}^{0}$ :

$$
\begin{aligned}
& {\left[\begin{array}{c}
w_{j} \\
w_{\mathcal{D}}
\end{array}\right]=\tilde{G}^{0}\left[\begin{array}{l}
w_{j} \\
w_{\mathcal{D}}
\end{array}\right]+\tilde{F}^{0}\left[\begin{array}{l}
r_{j}+v_{j} \\
r_{\mathcal{D}}+v_{\mathcal{D}} \\
r_{\mathcal{Z}}+v_{\mathcal{Z}}
\end{array}\right]} \\
& =(I-D)^{-1}\left(\tilde{G}^{0}-D\right)\left[\begin{array}{l}
w_{j} \\
w_{\mathcal{D}}
\end{array}\right]+(I-D)^{-1} \tilde{F}^{0}\left[\begin{array}{l}
r_{j}+v_{j} \\
r_{\mathcal{D}}+v_{\mathcal{D}} \\
r_{\mathcal{Z}}+v_{\mathcal{Z}}
\end{array}\right] .
\end{aligned}
$$

Both matrices in (22 have the same form as $\breve{G}^{0}$, and $\breve{F}^{0}$ in (10). Thus, by Proposition 1 , they are equal to $\breve{G}^{0}$ and $\breve{F}^{0}$.

\section{Proof of Lemma 1}

The following lemma is used in the proof. It can be proved using Mason's Rules [28], or as shown in Appendix A of [17].

Lemma 3: Consider a dynamic network with transfer matrix $G^{0}$ that satisfies all conditions of Assumption 1. Let $\mathcal{G}_{m n}^{0}$ be the $(m, n)$ th entry of $\left(I-G^{0}\right)^{-1}$. If all paths from $n$ to $m$ have a delay (are zero) then $\mathcal{G}_{m n}^{0}$ has a delay (is zero).

Now consider the proof of lemma 1.

Proof: Consider part 1. From Proposition 3, the transfer function of the $(m, n)$ th entry of $\breve{G}^{0}$ (where $m \neq n$ ) is

$$
\breve{G}_{m n}^{0}=\frac{1}{1-\tilde{G}_{m m}^{0}}\left(G_{m n}^{0}+\sum_{\ell_{1} \in \mathcal{Z}_{j}} \sum_{\ell_{2} \in \mathcal{Z}_{j}} G_{m \ell_{1}}^{0} \mathcal{G}_{\ell_{1} \ell_{2}}^{\mathcal{Z}} G_{\ell_{2} n}^{0}\right)
$$

where $\mathcal{G}_{\ell_{1} \ell_{2}}^{\mathcal{Z}}$ denotes the $\left(\ell_{2}, \ell_{1}\right)$ entry of $\left(I-G_{\mathcal{Z}}^{0}\right)^{-1}$. By Lemma 3 if every path from $\ell_{2}$ to $\ell_{1}$ passing only through nodes $w_{k}, k \in \mathcal{Z}_{j}$ has a delay then $\mathcal{G}_{\ell_{1} \ell_{2}}^{\mathcal{Z}}$ has a delay. Thus, if every path from $w_{k_{1}}$ to $w_{k_{2}}$ that passes only through nodes $w_{k}, k \in \mathcal{Z}_{j}$ has a delay, either $G_{m \ell_{1}}^{0}, \mathcal{G}_{\ell_{1} \ell_{2}}^{\mathcal{Z}}$, or $G_{\ell_{2} n}^{0}$ has a delay (for every $\ell_{1}$ and $\ell_{2}$ ). By (23) the statement of the lemma follows.

To show that $\breve{G}_{m n}^{0}=0$ when there is no path from $w_{m}$ to $w_{n}$ that passes through only nodes $w_{k}, k \in \mathcal{Z}_{j}$ follows the same reasoning, as does part 2 of the Lemma.

\section{E. Proof of Proposition 4}

Proof: From Algorithm 3 there are two ways that the transfer function $\breve{G}_{j i}^{0}$ can change to be different from $G_{j i}^{0}$ : in Steps 2c and 3. Using the same notation as that in Algorithm 3 , the proof will proceed by showing that Conditions (b) and (c) ensure that no change to $\breve{G}_{j i}^{(k)}$ occurs for all $k=1: d$ in Steps 2c and 3 respectively.

Start by investigating Step 2c. A change to $G_{j i}^{(k)}$ occurs if a path has been lifted in Step $2 \mathrm{a}$ and resulted in an edge from $w_{i}$ to $w_{j}$. By Condition (b) every path from $w_{i}$ to $w_{j}$ passes through a node $w_{n}, n \in \mathcal{D}_{j}$. Consequently, it will never occur at any iteration $k$ that a node $w_{n}$ is being removed that has an incoming edge from $w_{i}$ and an outgoing edge to $w_{j}$. Thus, there will never be parallel edges generated from $w_{i}$ to $w_{j}$ that must be merged in Step 2c.

Similarly, by Condition (c) every path from $w_{j}$ to $w_{j}$ passes through a node $w_{n}, n \in \mathcal{D}_{j}$. Consequently, it will never occur at any iteration $k$ of the algorithm that a node $w_{n}$ is being removed that has an incoming edge from $w_{j}$ and an outgoing edge to $w_{j}$. Thus there is never a self loop from $w_{j}$ to $w_{j}$ generated. Which means that the division in Step 3 will simply be a division by 1 .

\section{F. Proof of Theorem 1}

The following Lemma will be used to prove Theorem 1.

Lemma 4: Consider a vector of rational functions $\Delta X(q, \theta)=\left[\begin{array}{llll}\Delta X_{1}\left(q, \theta_{1}\right) & \cdots & \Delta X_{d}\left(q, \theta_{d}\right)\end{array}\right]^{T}$, where $\Delta X_{k}\left(q, \theta_{k}\right)=L_{k}\left(q, \theta_{k}\right)\left(X_{k}^{0}(q)-X_{k}\left(q, \theta_{k}\right)\right)$, where $L_{k}$ is a monic transfer function, $X_{k}^{0}$ is a transfer function and $X_{k}\left(\theta_{k}\right)$ is a transfer function parameterized as:

$$
X_{k}\left(\theta_{k}\right)=\frac{b_{0}^{k}+b_{1}^{k} q^{-1}+\cdots+b_{n_{b}}^{k} q^{-n_{b}}}{1+a_{1}^{k} q^{-1}+\cdots+a_{n_{a}}^{k} q^{-n_{a}}},
$$

where $\theta_{k}=\left[\begin{array}{llllll}b_{0}^{k} & \cdots & b_{n_{b}}^{k} & a_{1}^{k} & \cdots & a_{n_{a}}^{k}\end{array}\right]^{T}$. Suppose the parameterization is chosen such that for each $\Delta X_{k}\left(\theta_{k}\right)$, there exists a parameter vector $\theta^{*}$ such that $\Delta X\left(\theta^{*}\right)=0$. Consider a $(d \times d)$ power spectral density matrix $\Phi$. If $\Phi$ is positive definite for at least $n_{\theta}=n_{a}+n_{b}+1$ frequencies $\omega_{n}$, where $-\pi<\omega_{n} \leq \pi$ then

$\int_{-\pi}^{\pi} \Delta X\left(e^{j \omega}, \theta\right)^{T} \Phi(\omega) \Delta X\left(e^{-j \omega}, \theta\right) \mathrm{d} \omega=0 \Longrightarrow \Delta X_{k}(q, \theta)=0$ for $k=1, \ldots, d$.

For a proof see [33]. The proof of Theorem 1 now proceeds:

Proof: Consider the proof for Case 2 (Conditions (a) and (b) hold for only $\eta^{*}$ and $H_{j}^{-1}\left(q, \eta^{*}\right) p_{j}\left(t, \mathcal{D}_{j}\right)$ is white). The proof for Case 1 is not presented here since it follows the exact same line of reasoning, and is simpler than, that of Case 2. Since the noise model is independently parameterized from the module models, let $\eta$ denote the parameters associated with the noise model, and let $\theta$ denote the parameters associated with the modules.

For notational simplicity, let $H_{j}^{-1}\left(q, \eta^{*}\right) p_{j}\left(t, \mathcal{D}_{j}\right)$ be denoted as $s_{j}\left(t, \mathcal{D}_{j}\right)$. The reasoning will be split into two steps:

1. Show that if Conditions (a) and (b) hold at $\eta^{*}$, then the following bound on the objective function holds:

$$
\bar{V}(\theta) \geq \overline{\mathbb{E}}\left[\left(H_{j}^{-1}\left(q, \eta^{*}\right) p_{j}\left(t, \mathcal{D}_{j}\right)\right)^{2}\right] .
$$

2. Show that when equality holds it implies that $G_{j k}(q, \theta)=$ $\breve{G}_{j k}^{0}\left(q, \mathcal{D}_{j}\right), k \in \mathcal{D}_{j}$, and $F_{j k}(q, \theta)=\breve{F}_{j k}^{0}\left(q, \mathcal{D}_{j}\right), k \in \mathcal{P}_{j}$.

Step 1. From (14) and (6) it follows that

$$
\begin{array}{r}
\bar{V}(\theta, \eta)=\overline{\mathbb{E}}\left[\left(H _ { j } ^ { - 1 } ( q , \eta ) \left(w_{j}(t)-\sum_{k \in \mathcal{D}_{j}} G_{j k}(q, \theta) w_{k}^{(\mathcal{X})}(t)\right.\right.\right. \\
\left.\left.\left.-\sum_{k \in \mathcal{P}_{j}} F_{j k}(q, \theta) r_{k}(t)\right)\right)^{2}\right] .
\end{array}
$$


By (17) and (18) $w_{j}$ can be expressed in terms of $w_{k}, k \in \mathcal{D}_{j}$, $r_{k}, k \in \mathcal{P}_{j}$ and a residual, $p_{j}\left(t, \mathcal{D}_{j}\right)$ resulting in:

$$
\begin{aligned}
\bar{V}(\theta, \eta) & =\overline{\mathbb{E}}\left[\left(H _ { j } ^ { - 1 } ( q , \eta ) \left(\sum_{k \in \mathcal{D}_{j}} \Delta G_{j k}\left(q, \theta, \mathcal{D}_{j}\right) w_{k}^{(\mathcal{X})}(t)\right.\right.\right. \\
& \left.\left.\left.+\sum_{k \in \mathcal{P}_{j}} \Delta F_{j k}\left(q, \theta, \mathcal{D}_{j}\right) r_{k}(t)+p_{j}\left(t, \mathcal{D}_{j}\right)\right)\right)^{2}\right]
\end{aligned}
$$

where $\Delta G_{j k}\left(q, \theta, \mathcal{D}_{j}\right)=\breve{G}_{j k}^{0}\left(q, \mathcal{D}_{j}\right)-G_{j k}(q, \theta)$, and $\Delta F_{j k}\left(q, \theta, \mathcal{D}_{j}\right)=\breve{F}_{j k}^{0}\left(q, \mathcal{D}_{j}\right)-F_{j k}(q, \theta)$. If (25) is evaluated at $\eta^{*}$, the third term is equal to $s_{j}$.

By Conditions (a) and (b) $s_{j}$ is uncorrelated to the first two terms in the expression. Moreover, since $s_{j}$ is white, it is also uncorrelated to delayed versions of itself which means that $\mathbb{E}\left[\Delta H_{j}(q, \eta) s_{j}(t) \cdot s_{j}(t)\right]=0$ where $\Delta H_{j}\left(q, \eta, \mathcal{D}_{j}\right)=$ $H_{j}\left(q, \eta^{*}\right)-H_{j}(q, \eta)$ (the expression holds since $H_{j}$ is monic, and thus $\Delta H_{j}$ has a delay).

Using this fact to simplify (25) results in

$$
\begin{gathered}
\bar{V}(\theta, \eta)=\overline{\mathbb{E}}\left[s_{j}^{2}\left(\mathcal{D}_{j}\right)\right]+\overline{\mathbb{E}}\left[H _ { j } ^ { - 1 } ( \eta ) \left(\sum_{k \in \mathcal{D}_{j}} \Delta G_{j k}\left(\theta, \mathcal{D}_{j}\right) w_{k}^{(\mathcal{X})}\right.\right. \\
\left.\left.+\sum_{k \in \mathcal{P}_{j}} \Delta F_{j k}\left(\theta, \mathcal{D}_{j}\right) r_{k}+\Delta H_{j}\left(\eta, \mathcal{D}_{j}\right) s_{j}\left(\mathcal{D}_{j}\right)\right)^{2}\right] .
\end{gathered}
$$

The first term of $\bar{V}(\theta, \eta)$ is not a function of $\theta$ or $\eta$, proving that $\bar{V}(\theta, \eta) \geq \overline{\mathbb{E}}\left[s_{j}^{2}\left(t, \mathcal{D}_{j}\right)\right]$ as desired.

Step 2. Now it is shown that

$\bar{V}(\theta, \eta)=\overline{\mathbb{E}}\left[s_{j}^{2}\left(t, \mathcal{D}_{j}\right)\right] \Rightarrow\left\{\begin{array}{l}G_{j k}(q, \theta)=\breve{G}_{j k}^{0}\left(q, \mathcal{D}_{j}\right), k \in \mathcal{D}_{j} \\ F_{j k}(q, \theta)=\breve{F}_{j k}^{0}\left(q, \mathcal{D}_{j}\right), k \in \mathcal{P}_{j} \\ H_{j}(q, \eta)=H_{j}\left(q, \eta^{*}\right)\end{array}\right.$

Consider the equation $\bar{V}(\theta, \eta)=\overline{\mathbb{E}}\left[s_{j}^{2}\left(t, \mathcal{D}_{j}\right)\right]$. From (26) using Parseval's theorem, this results in:

$$
\frac{1}{2 \pi} \int_{-\pi}^{\pi} \Delta X\left(e^{j \omega}, \theta\right) \Phi(\omega) \Delta X^{T}\left(e^{-j \omega}, \theta\right) \mathrm{d} \omega=0,
$$

for all $\omega \in[-\pi, \pi]$, where

$$
\begin{aligned}
\Delta X & =H_{j}^{-1}\left[\Delta G_{j k_{1}} \cdots \Delta G_{j k_{n}} \Delta F_{j m_{1}} \cdots \Delta F_{j m_{\ell}} \Delta H_{j}\right], \\
\Phi(\omega) & =\left[\begin{array}{ccc}
\Phi_{w_{\mathcal{D}}}(\omega) & \Phi_{w_{D} r_{\mathcal{P}}}(\omega) & \Phi_{w_{\mathcal{D}} s_{j}}(\omega) \\
\Phi_{r_{\mathcal{P}} w_{\mathcal{D}}}(\omega) & \Phi_{r_{\mathcal{P}}}(\omega) & \Phi_{r_{\mathcal{P}} s_{j}}(\omega) \\
\Phi_{s_{j} \omega_{\mathcal{D}}}(\omega) & \Phi_{s_{j} r_{\mathcal{P}}}(\omega) & \Phi_{s_{j}}(\omega)
\end{array}\right],
\end{aligned}
$$

where $k_{*} \in \mathcal{D}_{j}, m_{*} \in \mathcal{P}_{j}$ and $\Phi_{* *}(\omega)$ are the (cross) power spectral densities of the denoted variables. Recall from (18) that $w_{j}$ can be expressed in terms of $w_{k}^{(\mathcal{X})}, k \in \mathcal{D}_{j}, r_{k}, k \in \mathcal{P}_{j}$ and $p_{j}$. By rearanging (18) an expression for $s_{j}$ is

$$
s_{j}=H_{j}^{0^{-1}}\left(w_{j}-\sum_{k \in \mathcal{D}_{j}} \breve{G}_{j k}^{0} w_{k}^{(\mathcal{X})}-\sum_{k \in \mathcal{P}_{j}} \breve{F}_{j k}^{0} r_{k}\right)
$$

Consequently, (28) can be expressed as $J \Phi_{w} J^{H}$, where

$$
J=\left[\begin{array}{ccc}
I & 0 & 0 \\
0 & I & 0 \\
-\breve{G}_{j \mathcal{D}}^{0} & -\breve{F}_{j \mathcal{D}}^{0} & 1
\end{array}\right]
$$

$\Phi_{w} \quad$ is the power spectral density of $\left[\begin{array}{llllll}w_{k_{1}} & \cdots & w_{k_{n}} & r_{m_{1}} & \cdots & r_{m_{\ell}} \\ w_{j}\end{array}\right]$, and $(\cdot)^{H}$ denotes conjugate transpose. Because $J$ is full rank for all $\omega$, and $\Phi_{w}$ is full rank for at least $n_{\theta}$ frequencies (by the statement of the theorem) it follows that $\Phi$ in (27) is full rank for at least $n_{\theta}$ frequencies. Because $\Phi(\omega)$ is positive definite for at least $n_{\theta}$ frequencies, and the parameterization is chosen flexible enough, it follows from Lemma 4 that $\Delta X=0$. By the definition of $\Delta X$ it follows that (27) implies $G_{j k}\left(q, \theta^{*}\right)=\breve{G}_{j k}\left(q, \mathcal{D}_{j}\right), k \in \mathcal{D}_{j}$, $F_{j k}\left(q, \theta^{*}\right)=\breve{G}_{j k}\left(q, \mathcal{D}_{j}\right), k \in \mathcal{P}_{j}$, and $H_{j}\left(q, \theta^{*}\right)=H_{j}\left(q, \eta^{*}\right)$ as desired.

\section{G. Proof of Proposition 5}

Proof: The proof proceeds by showing that Conditions (a) and (b) hold at $\eta^{*}$, and that $H_{j}^{-1}\left(\eta^{*}\right) p_{j}$ is white noise. By Condition (d), $p_{j}$ is not a function of any $r$ terms, and thus from (17) it follows that $p_{j}=\breve{v}_{j}$. Recall from (10) that the equation defining the immersed network is $w=\breve{G}^{0} w+\breve{F}^{0} r+\breve{v}$ where $w=\left[\begin{array}{ll}w_{j} & w_{\mathcal{D}}\end{array}\right]^{T}, r=\left[\begin{array}{lll}r_{j} & r_{\mathcal{D}} & r_{\mathcal{Z}}\end{array}\right]^{T}$ and $\breve{v}$ is defined in (12). Consequently, $w_{k}$ can be expressed as

$$
w_{k}=\breve{\mathcal{G}}_{k j}^{0}\left(\breve{v}_{j}+r_{j}+\breve{F}_{j \mathcal{z}}^{0} r_{\mathcal{Z}}\right)+\sum_{n \in \mathcal{D}_{j}} \breve{\mathcal{G}}_{k n}^{0}\left(\breve{v}_{n}+r_{n}+\breve{F}_{n \mathcal{Z}}^{0} r_{\mathcal{Z}}\right)
$$

where $\breve{\mathcal{G}}_{j k}^{0}$ denotes the $(j, k)$ entry of $\left(I-\breve{G}^{0}\right)^{-1}$. Using this expression for $w_{k}$, Condition (a) of Theorem 1 can be expressed as:

$$
\begin{gathered}
\overline{\mathbb{E}}\left[H_{j}^{-1}\left(q, \eta^{*}\right) p_{j}(t) \cdot \Delta G_{j k}\left(q, \mathcal{D}_{j}, \theta\right) w_{k}(t)\right]=\overline{\mathbb{E}}\left[H_{j}^{-1}\left(q, \eta^{*}\right) \breve{v}_{j}(t)\right. \\
\left.\cdot \Delta G_{j k}\left(q, \mathcal{D}_{j}, \theta\right) \sum_{n \in \mathcal{D}_{j} \cup\{j\}} \breve{\mathcal{G}}_{k n}^{0}(q)\left(\breve{v}_{n}(t)+r_{n}(t)+\breve{F}_{n \mathcal{Z}}^{0}(q) r_{\mathcal{Z}}(t)\right)\right] .
\end{gathered}
$$

By Assumption 1 every $v_{k}$ is uncorrelated to every external variable. Moreover, by Condition (a) $\breve{v}_{j}$ is uncorrelated to the other noise terms in the immersed network, and so the above equation can be simplified:

$$
\begin{aligned}
& \overline{\mathbb{E}}\left[H_{j}^{-1}\left(q, \eta^{*}\right) p_{j}(t) \cdot \Delta G_{j k}\left(q, \mathcal{D}_{j}, \theta\right) w_{k}(t)\right] \\
& \quad=\overline{\mathbb{E}}\left[H_{j}^{-1}\left(q, \eta^{*}\right) \breve{v}_{j}(t) \cdot \Delta G_{j k}\left(q, \mathcal{D}_{j}, \theta\right) \breve{\mathcal{G}}_{k j}^{0}(q) \breve{v}_{j}(t)\right]
\end{aligned}
$$

By Lemma 3 (in Appendix X-D) the transfer function $\breve{\mathcal{G}}_{k j}^{0}$ has a delay if every path (in the immersed network) from $w_{j}$ to $w_{k}$ has a delay. It follows by Condition (b) that either $\breve{\mathcal{G}}_{k j}^{0}$ or $\breve{G}_{j k}^{0}$ (or both) has a delay. By Condition (c) it follows that either $\breve{\mathcal{G}}_{k j}^{0}$ or $\Delta G_{j k}\left(q, \mathcal{D}_{j}, \theta\right)$ (or both) has a delay. The result is that $\Delta G_{j k}\left(q, \mathcal{D}_{j}, \theta\right) \breve{\mathcal{G}}_{k j}^{0} \breve{v}_{j}$ is a function of only delayed versions of $\breve{v}_{j}$ (and thus delayed versions of $\breve{e}_{j}$, where $\breve{e}_{j}$ is the whitened version of $\breve{v}_{j}$ as defined in (13)). Thus it follows that

$$
\begin{aligned}
\overline{\mathbb{E}}\left[H_{j}^{-1}\left(q, \eta^{*}\right) p_{j}(t)\right. & \left.\cdot \Delta G_{j k}\left(q, \mathcal{D}_{j}, \theta\right) w_{k}(t)\right] \\
& =\overline{\mathbb{E}}\left[\breve{e}_{j}(t) \cdot \Delta G_{j k}\left(q, \mathcal{D}_{j}, \theta\right) \breve{\mathcal{G}}_{k j}^{0}(q) \breve{v}_{j}(t)\right]=0
\end{aligned}
$$

which means that the Condition (a) of Theorem 1 holds.

Since $p_{j}=\breve{v}_{j}$, and by Assumption 1, all $v$ 's are uncorrelated to all $r$, it follows that Condition (b) holds as well.

\section{H. Proof of Proposition 6}

Proof: The following reasoning will show that $\overline{\mathbb{E}}\left[\breve{v}_{j}(t)\right.$. $\left.\breve{v}_{k}(t-\tau)\right]=0$ for all $\tau$. From (12),

$$
\begin{aligned}
\overline{\mathbb{E}}\left[\breve{v}_{j}(t) \cdot \breve{v}_{k}(t-\tau)\right] & =\overline{\mathbb{E}}\left[\left(v_{j}(t)+\breve{F}_{j \mathcal{Z}}^{0}(q) v_{\mathcal{Z}}(t)\right)\right. \\
\cdot & \left.\left(v_{k}(t-\tau)+\breve{F}_{k \mathcal{Z}}^{0}(q) v_{\mathcal{Z}}(t-\tau)\right)\right] .
\end{aligned}
$$


Consider the following three facts. First, by Condition (a), $v_{j}$ is uncorrelated to all $v_{k}, k \in \mathcal{D}_{j}$. Secondly,

$$
\overline{\mathbb{E}}\left[v_{j}(t) \cdot \breve{F}_{k n}^{0}(q) v_{n}(t-\tau)\right]=0, \forall \tau \text {, and } \forall n \in \mathcal{Z}_{j}
$$

by the following reasoning. Either one of the conditions holds:

- There is a path from $v_{n}, n \in \mathcal{Z}_{j}$ to $w_{k}$ that passes only through nodes $w_{k}, k \in \mathcal{Z}_{j}$. In this case, by Condition (a) $v_{j}$ is uncorrelated to $v_{n}$.

- There is no path from $v_{n}, n \in \mathcal{Z}_{j}$ to $w_{k}$. In this case, by Lemma $1, \breve{F}_{k n}^{0}$ is zero. Consequently, $\overline{\mathbb{E}}\left[v_{j}(t)\right.$. $\left.\breve{F}_{k n}(q) v_{n}(t)\right]=0$.

Thirdly, by the same reasoning and by Condition (b), $\overline{\mathbb{E}}\left[v_{k}(t)\right.$. $\left.F_{j z}(q) v_{\mathcal{z}}(t-\tau)\right]=0$ for all $\tau$. Consequently, (30) can be simplified to:

$$
\overline{\mathbb{E}}\left[\breve{v}_{j}(t) \breve{v}_{k}(t-\tau)\right]=\overline{\mathbb{E}}\left[\breve{F}_{j \mathcal{Z}}^{0}(q) v_{\mathcal{Z}}(t) \cdot \breve{F}_{k \mathcal{Z}}^{0}(q) v_{\mathcal{Z}}(t-\tau)\right] .
$$

By Parseval's Theorem this equation can be expressed as

$$
\overline{\mathbb{E}}\left[\breve{v}_{j}(t) \breve{v}_{k}(t-\tau)\right]=\frac{1}{2 \pi} \int_{-\pi}^{\pi} \breve{F}_{j \mathcal{Z}}^{0}\left(e^{j \omega}\right) \Phi_{v_{\mathcal{Z}}}(\omega) \breve{F}_{k \mathcal{Z}}^{0^{T}}\left(e^{-j \omega}\right) e^{j \omega \tau} \mathrm{d} \omega .
$$

By Condition (c), $\Phi_{v_{z}}$ is diagonal, and so

$$
\overline{\mathbb{E}}\left[\breve{v}_{j}(t) \breve{v}_{k}(t-\tau)\right]=\frac{1}{2 \pi} \int_{-\pi}^{\pi} \sum_{\ell \in \mathcal{Z}_{j}} \breve{F}_{j \ell}\left(e^{j \omega}\right) \breve{F}_{k \ell}\left(e^{-j \omega}\right) e^{j \tau \omega} \phi_{\ell}(\omega) \mathrm{d} \omega
$$

where $\phi_{\ell}$ is the power spectral density of $v_{\ell}$. By Lemma 1 the transfer function $\breve{F}_{j k}^{0}$ is zero if there is no path from $v_{k}$ to $w_{j}$ that passes only through nodes $w_{k}, k \in \mathcal{Z}_{j}$. Consequently, by Condition (d) for each $\ell, \ell \in \mathcal{Z}_{j}$, either $\breve{F}_{j \ell}^{0}$ or $\breve{F}_{k \ell}^{0}$ (or both) are equal to zero. Consequently, $\overline{\mathbb{E}}\left[\breve{v}_{j}(t) \breve{v}_{k}(t-\tau)\right]$ is equal to zero for al $\tau$, and for all $k \in \mathcal{D}_{j}$.

\section{Proof of Proposition 8}

Proof: The proof proceeds by showing that Case 1 of Theorem 1 holds. The predictor inputs $w_{k}^{\left(\tau_{j}\right)}, k \in \mathcal{D}_{j}$ and $r_{k}$, $k \in \mathcal{P}_{j}$ are functions of all $r_{k}, k \in\left\{\mathcal{T}_{j}\right\}$, except those $r_{k}$ for which there is no path $r_{k}$ to $w_{j}$ (the projection onto this external variable is zero in this case). Thus it is sufficient to show that the optimal output error residual of $w_{j}$ is not correlated to these $r$ 's. From (17) $p_{j}$ is equal to

$$
\begin{aligned}
p_{j}\left(t, \mathcal{D}_{j}\right)= & \breve{F}_{j j}^{0}\left(q, \mathcal{D}_{j}\right) r_{j}(t)+\breve{v}_{j}(t)+\sum_{k \in\left(\mathcal{Z}_{j} \cap \mathcal{R}_{j}\right) \backslash \mathcal{P}_{j}} \breve{F}_{j k}^{0}\left(q, \mathcal{D}_{j}\right) r_{k}(t) \\
& +\sum_{k \in \mathcal{D}_{j}} \breve{G}_{j k}^{0}\left(q, \mathcal{D}_{j}\right) w_{k}^{\left(\perp \mathcal{T}_{j}\right)}(t) .
\end{aligned}
$$

By Assumption 1 all $r$ 's are uncorrelated to all $v$ 's. Thus, only the $r$ terms in $p_{j}$ could cause a correlation between $p_{j}$ and the predictor inputs. In particular, it must be shown that $p_{j}$ is not a function of any $r_{k}, k \in \mathcal{T}_{j}$.

Split the variables in $\mathcal{T}_{j}$ into two categories: the $r_{k}$ 's for which at least one path from $r_{k}$ to $w_{j}$ passes only through nodes in $\mathcal{Z}_{j}$, and the $r_{k}$ 's for which all paths from $r_{k}$ to $w_{j}$ pass through at least one node $w_{k}, k \in \mathcal{D}_{j}$. By construction, all $r_{k}$ 's that are in the first category are in $\mathcal{P}_{j}$. Since no variable $r_{k} \in \mathcal{P}_{j}$ appears in $p_{j}$ (see (32) none of the variables in the first category appear in the expression for $p_{j}$.
By Lemma 1 it follows that for all $r_{k}$ in the second category $\breve{F}_{j k}^{0}$ is zero. Thus, from (32) it follows that no $r_{k}$ term in the first category will appear in the expression for $p_{j}$.

Thus, $p_{j}$ is not a function of any $r_{k}, k \in \mathcal{T}_{j}$. Consequently, $p_{j}$ is uncorrelated to the predictor inputs, and the conditions of Theorem 1 are satisfied.

Lastly, to satisfy all the conditions of Theorem 1 we must show that the power spectral density $\Phi$ of

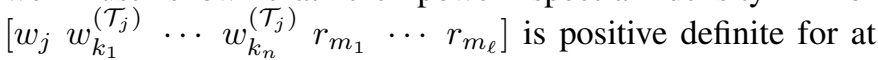
least $n_{\theta}$ frequencies. By (18) $p_{j}$ can be expressed as a function of $\left.w_{k}^{\left(\mathcal{T}_{j}\right.}\right), k \in \mathcal{D}_{j}$, and $r_{k}, k \in \mathcal{P}_{j}$ and $p_{j}$. It has allready been shown that $p_{j}$ is uncorrelated to all the predictor inputs. Consequently, the power spectral density $\Phi$ is equal to

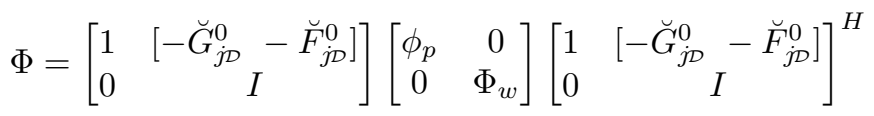

where $\phi_{p}$ is the power spectral density of $p_{j}$ and $\Phi_{w}$ is the power spectral density of $\left[\begin{array}{llllll}w_{k_{1}}^{\left(\mathcal{T}_{j}\right)} & \cdots & w_{k_{n}}^{\left(\mathcal{T}_{j}\right)} & r_{m_{1}} & \cdots & r_{m_{\ell}}\end{array}\right]$ (which is positive definite at $n_{\theta}$ frequencies). Because the first (and last) matrices are full rank for all $\omega$ it follows that $\Phi$ is full rank for at least $n_{\theta}$ frequencies. Consequently all the conditions of Case 1 of Theorem 1 are satisfied.

\section{REFERENCES}

[1] C. W. J. Granger, "Testing for causality; a personal viewpoint," Journal for Economic Dynamics and Control, pp. 329-352, 1980.

[2] P. E. Caines and C. W. Chan, "Feedback between stationary stochastic processes," IEEE Transactions on Automatic Control, vol. 20, no. 4, pp. 498-508, 1975.

[3] M. R. Gevers and B. D. O. Anderson, "Representing of jointly stationary stochastic feedback processes," International Journal of Control, vol. 33, no. 5, pp. 777-809, 1981.

[4] D. Materassi and G. Innocenti, "Topological identification in networks of dynamical systems," IEEE Transactions on Automatic Control, vol. 55, no. 8, pp. 1860-1871, 2010.

[5] D. Materassi and M. V. Salapaka, "On the problem of reconstructing an unknown topology via locality properties of the Wiener filter," IEEE Transactions on Automatic Control, vol. 57, no. 7, pp. 1765-1777, July 2012.

[6] A. Seneviratne and V. Solo, "Topology identification of a sparse dynamic network," in Proceedings of the 51st IEEE Conference on Decision and Control (CDC), Maui, HI, USA, Dec 2012, pp. 1518-1523.

[7] Y. Yuan, G. Stan, S. Warnick, and J. Goncalves, "Robust dynamical network structure reconstruction," Automatica, vol. 47, no. 6, pp. 1230 $-1235,2011$

[8] J. Friedman, T. Hastie, and R. Tibshirani, "Applications of the lasso and grouped lasso to the estimation of sparse graphical models," 2010, unpublished. [Online]. Available: http://wwwstat.stanford.edu/tibs/ftp/ggraph.pdf

[9] R. Tibshirani, "Regression shrinkage and selection via the lasso," Journal of the Royal Statistical Society, Series B, vol. 58, pp. 267-288, 1994.

[10] B. M. Sanandaji, T. L. Vincent, and M. B. Wakin, "A review of sufficient conditions for structure identification in interconnected systems," in Proceedings of the 16th IFAC Symposium on System Identification, Brussels, Belgium, July 2012, pp. 1623-1628.

[11] A. Chuiso and G. Pillonetto, "A Bayesian approach to sparse dynamic network identification,” Automatica, vol. 48, pp. 1553-1565, 2012.

[12] R. Fraanje and M. Verhaegen, "A spatial canonical approach to multidimensional state-space identification for distributed parameter systems," in Proceedings of the Fourth International Workshop on Multidimensional Systems, July 2005, pp. 217-222.

[13] M. Ali, S. S. Chughtai, and H. Werner, "Identification of spatially interconnected systems," in Proceedings of the 48th IEEE Conference on Decision and Control (CDC), held jointly with the 28th Chinese Control Conference (CCC), Shanghai, China, Dec 2009, pp. 7163-7168.

[14] A. Sarwar, P. G. Voulgaris, and S. M. Salapaka, "System identification of spatiotemporally invariant systems," in Proceedings of the American Control Conference (ACC), Baltimore, MD, USA, June 2010, pp. 2947 2952. 
[15] P. Torres, J.-W. van Wingerden, and M. Verhaegen, "Hierarchical subspace identification of directed acyclic graphs," International Journal of Control, vol. 88, no. 1, pp. 123-137, 2015.

[16] A. Haber and M. Verhaegen, "Moving horizon estimation for largescale interconnected systems," IEEE Transactions on Automatic Control, vol. 58, no. 11, pp. 2834-2847, Nov 2013.

[17] P. M. J. Van den Hof, A. Dankers, P. S. C. Heuberger, and X. Bombois, "Identification of dynamic models in complex networks with prediction error methods - basic methods for consistent module estimates," Automatica, vol. 49, pp. 2994-3006, Oct. 2013.

[18] A. Dankers, P. M. J. Van den Hof, X. Bombois, and P. S. C. Heuberger, "Predictor input selection for two stage identification in dynamic networks," in Proceedings of the European Control Conference (ECC), Zürich, Switzerland, Jul. 2013, pp. 1422-1427.

[19] A. Dankers, P. M. J. Van den Hof, and P. S. C. Heuberger, "Predictor input selection for direct identification in dynamic networks," in Proceedings of the 52nd IEEE Conference on Decision and Control (CDC), Florence, Italy, December 2013, pp. 4541-4546.

[20] M. Araki and M. Saeki, "A quantitative condition for the well-posedness of interconnected dynamical systems," IEEE Transactions on Automatic Control, vol. 28, no. 5, pp. 625-637, May 1983.

[21] L. Ljung, System Identification. Theory for the User, 2nd ed. Prentice Hall, 1999.

[22] P. M. J. Van den Hof and R. Schrama, "An indirect method for transfer function estimation from closed loop data," Automatica, vol. 29, no. 6, pp. $1523-1527,1993$.

[23] P. M. J. Van den Hof, "Closed-loop issues in system identification," Annual Reviews in Control, vol. 22, pp. 173-186, 1998.

[24] U. Forssell and L. Ljung, "Closed-loop identification revisited," Automatica, vol. 35, pp. 1215-1241, 1999.

[25] J. Gonçalves and S. Warnick, "Necessary and sufficient conditions for dynamical structure reconstruction of LTI networks," IEEE Transactions on Automatic Control, vol. 53, no. 7, pp. 1670-1674, Aug. 2008.

[26] D. C. Youla, "On the factorization of rational matrices," IRE Transactions on Information Theory, vol. 7, pp. 172-189, Jul. 1961.

[27] M. A. Langston and B. C. Plaut, "On algorithmic applications of the immersion order," Discrete Mathematics, vol. 182, pp. 191-196, 1998.

[28] S. J. Mason, "Feedback theory - some properties of signal flow graphs," Proceedings of The IRE, pp. 1144-1156, Sep. 1953

[29] — "Feedback theory - further properties of signal flow graphs," Proceedings of The IRE, pp. 920-926, Jul. 1956.

[30] T. Söderström and P. Stoica, System Identification, ser. Systems and Control Engineering. Hertfordshire, UK: Prentice Hall, 1989.

[31] J. Pearl, "Causal inference in statistics: An overview," Statistics Surveys, vol. 3, pp. 96-146, 2009.

[32] M. Gevers, A. S. Bazanella, X. Bombois, and L. Mišković, "Identification and the information matrix: How to get just sufficiently rich?" IEEE Transactions on Automatic Control, vol. 54, pp. 2828-2840, 2009.

[33] A. Dankers, "System identification in dynamic networks," Ph.D. dissertation, Delft University of Technology, 2014.

[34] A. Dankers, P. M. J. Van den Hof, X. Bombois, and P. S. C. Heuberger "Errors in variables identification in dynamic networks by an instrumental variable approach," in Proceedings of 19th IFAC World Congress, Cape Town, South Africa, 2014, pp. 2335- 2340.

[35] R. Diestel, Graph Theory, ser. Graduate Texts in Mathematics. Springer-Verlag, 1997, no. 173.

[36] A. Kanevsky, "Finding all minimum-size separating vertex sets in a graph," Networks, vol. 23, pp. 533-541, 1993.

[37] P. S. C. Heuberger, P. M. J. Van den Hof, and B. Wahlberg, Eds., Modelling and Identification with Rational Orthogonal Basis Functions. London: Springer-Verlag, 2005.

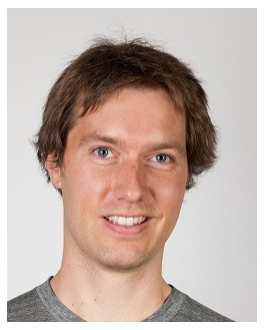

Arne Dankers $\left(S^{\prime} 10-\mathrm{M}^{\prime} 15\right)$ received B.Sc. and M.Sc. degrees from the Department of Electrical and Computer Engineering at the University of Calgary, and a Ph.D. degree from the Delft Center for Systems and Control at the Delft University of Technology. Currently he holds a Post-Doc position at the University of Calgary in partnership with Hifi Engineering Inc.. His current research interests include system identification and dynamic networks, acoustic modelling and leak detection in pipelines. $\mathrm{He}$ is the winner of the best student paper award of the 2013 European Control Conference.

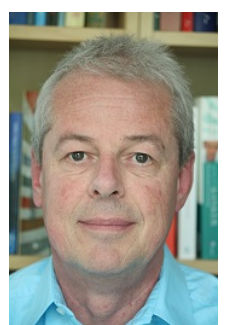

Paul M. J. Van den Hof $\left(\mathrm{S}^{\prime} 85-\mathrm{M}^{\prime} 88-\mathrm{SM}^{\prime} 95\right.$ - $\mathrm{F}^{\prime} 08$ ) received the M.Sc. and Ph.D. degrees in electrical engineering from Eindhoven University of Technology, Eindhoven, The Netherlands, in 1982 and 1989, respectively. In 1986 he moved to Delft University of Technology, where he was appointed as Full Professor in 1999. Since 2003, he has been founding co-director of the Delft Center for Systems and Control (DCSC), with appointments in the faculty of Mechanical, Maritime, and Materials Engineering, and the faculty of Applied Sciences. As of 2011, he is a Full Professor in the Electrical Engineering Department, Eindhoven University of Technology. His research interests include issues of system identification, identification for control, and model-based control and optimization, with applications in industrial process control systems, including petroleum reservoir engineering systems, and high-tech systems. Prof. Van den Hof is an IFAC Fellow. He has been a member of the IFAC Council (1999-2005), the Board of Governors of IEEE Control Systems Society (2003-2005), and an Associate Editor and Editor of Automatica (1992-2005). He was General Chair of the 13th IFAC Symposium on System Identification, held in Rotterdam, The Netherlands, in 2003.

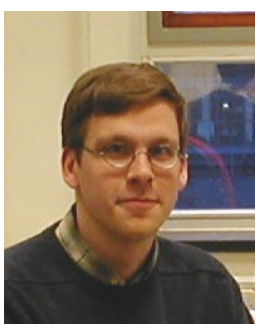

Xavier Bombois was born in Brussels in 1974. $\mathrm{He}$ received the Electrical Engineering and Ph.D. degrees from the Universite Catholique de Louvain (Belgium) in 1997 and 2000, respectively. In 2000, he moved to Delft University of Technology (The Netherlands) where he was appointed as an Assistant Professor at the Delft Center for Systems and Control. Since February 2015, he is a CNRS Research Director at Laboratoire Ampère (Ecole Centrale de Lyon, Ecully, France) His main interests are identification for control, prediction error identification, optimal experiment design uncertainty bounding and robust control. Since 2012, Xavier Bombois is an Associate Editor for the IFAC journal Control Engineering Practice and for the Conference Editorial Board of the IEEE Control Systems Society.

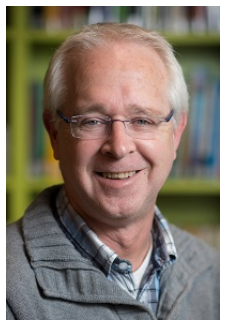

Peter S. C. Heuberger was born in Maastricht, The Netherlands, in 1957. He obtained the M.Sc. degree in Mathematics from the University of Groningen in 1983, and the Ph.D. degree from the Mechanical Engineering Department of Delft University of Technology in 1991. From 1991 to 2005 he was with the Dutch National Institute for Public Health and the Environment (RIVM) and from 2005 to 2007 with the Dutch Environmental Assessment Agency (MNP). From 1996 to 2007 he also held a parttime position at Delft University of Technology, in the Mechanical Engineering Systems and Control Group and the Signals, Systems and Control Group of the Department of Applied Physics. From 2007 to 2012 he was assistant professor at the Delft Center for Systems and Control. Since 2012 he is with Eindhoven University of Technology, where he is managing director of the automotive systems design program. His research interests are in issues of automotive systems, system identification, uncertainty, model reduction and in the theory and application of orthogonal basis functions. 